\title{
Robust stabilization of discrete-time periodic linear systems for tracking and disturbance rejection
}

published in Math. Control Signals Syst. 28(2016), pp.1-34, DOI 10.1007/s00498-016-0171-8

\author{
H. Bourlès, U. Oberst ${ }^{\dagger}$
}

July 11,2016

\begin{abstract}
In analogy to the Kučera-Youla parametrization we construct and parametrize all stabilizing controllers of a stabilizable linear periodic discrete-time input/output system, the plant. We establish a necessary and sufficient algebraic condition for the existence of controllers among these for which the output of the plant tracks a given reference signal in spite of disturbance signals on the input and the output of the plant. With a minor additional assumption the tracking stabilizing controllers are robust. As in the linear time-invariant (LTI) case the reference and disturbance signals are assumed to be generated by an autonomous system. Our results are the analogues for periodic behaviors of the corresponding LTI results of Vidyasagar. A completely different approach to stabilization and control of discrete periodic systems was developed by Bittanti and Colaneri. We derive a categorical duality between periodic behaviors over the time-axis of natural numbers and finitely generated modules over a suitable noncommutative ring of difference operators and use this for the proof of the main stabilization and control results. Morita's theory of equivalences between module categories is employed as an essential algebraic tool. All results of the paper are constructive.
\end{abstract}

AMS-classification: 93D15, 93D09, 93C55, 93C35, 93B25

Key-words: periodic behavior, stabilizing controller, tracking, disturbance rejection, robustness, Morita equivalence

\section{Introduction}

In analogy to the Kučera-Youla parametrization we construct and parametrize all stabilizing controllers of a stabilizable linear $N$-periodic $(N>0)$ discrete-time input/output (IO) system, the plant (Thm. 5.3). We establish a necessary and sufficient algebraic condition for the existence of controllers among these, for which the output of the plant tracks a given reference signal in spite of disturbance signals on the input and the output of the plant (Thms. 6.1, 6.2). With a minor additional assumption the tracking

\footnotetext{
*SATIE, ENS Cachan/CNAM, 61 Avenue President Wilson, F-94230, Cachan, France. email: henri.bourles@satie.ens-cachan.fr

${ }^{\dagger}$ Institut für Mathematik, Universität Innsbruck, Technikerstrasse 13, A-6020 Innsbruck, Austria. email: ulrich.oberst@uibk.ac.at
} 
stabilizing controllers are robust (Thm. 6.4). As in the linear time-invariant (LTI) case the reference and disturbance signals are assumed to be generated by an autonomous system. Our results are the analogues for periodic behaviors of the corresponding LTI results of Vidyasagar $[16, \S \S 5.1,5.2,5.7,7.5]$. They solve open problems that were raised in $[1, \S 7]$.

In contrast to [11], [1] and [5] and in accordance with [9], [2] and [16] (in the LTI case) we consider $N$-periodic systems on the time-axis $\mathbb{N} \ni t$ of natural numbers and not on $\mathbb{Z}$. A periodic system is a linear time-varying (LTV) system whose coefficient functions $a$ are $N$-periodic, i.e., satisfy $a(t+N)=a(t)$ for $t \in \mathbb{N}$. If $\mathbb{F}$ denotes any field or, in Sections 5 and 6, the field $\mathbb{R}$ or $\mathbb{C}$ of real or complex numbers, the coefficient functions form the commutative algebra $\mathbb{F}^{\mathbb{Z} / \mathbb{Z} N}$ of functions from $\mathbb{Z} / \mathbb{Z} N$ to $\mathbb{F}$ where we pose $a(t):=a(t+\mathbb{Z} N)$ for $t \in \mathbb{N}$. The monoid $\mathbb{N}$ acts on $a \in \mathbb{F}^{\mathbb{Z} / \mathbb{Z} N}$ via algebra isomorphisms by $(j \circ a)(t+\mathbb{Z} N):=a(j+t+\mathbb{Z} N)$. This action gives rise to the noncommutative skew-polynomial algebra of difference operators, cf. [5, (25)],

$$
\mathbf{A}:=\mathbb{F}^{\mathbb{Z} / \mathbb{Z} N}[q ; \circ]=\oplus_{j=0}^{\infty} \mathbb{F}^{\mathbb{Z} / \mathbb{Z} N} q^{j} \text { with } q^{j} a=(j \circ a) q^{j}, j \in \mathbb{N}, a \in \mathbb{F}^{\mathbb{Z} / \mathbb{Z} N} .
$$

The most general and standard signal space for one-dimensional discrete systems theory is the space

$$
W:=\mathbb{F}^{\mathbb{N}}:=\left\{w=(w(t))_{t \in \mathbb{N}}: \mathbb{N} \rightarrow \mathbb{F}, t \mapsto w(t)\right\}
$$

of sequences or functions from $\mathbb{N}$ to $\mathbb{F}$. The components of the error signals in the stabilization theory $(\mathbb{F}=\mathbb{R}, \mathbb{C})$ are, however, much more special and indeed exponentially stable and, in particular, belong to the Banach spaces

$$
\ell^{\infty}=\left\{w \in \mathbb{F}^{\mathbb{N}} ; \sup _{t \in \mathbb{N}}|w(t)|<\infty\right\} \text { and } \ell^{p}:=\left\{w \in \mathbb{F}^{\mathbb{N}} ; \sum_{t \in \mathbb{N}}|w(t)|^{p}<\infty\right\}
$$

for $p \in \mathbb{N}, p>0$. The proper and stable transfer matrix of the constructed closed loop behavior acts via convolution on vectors with entries in $\mathbb{F}^{\mathbb{N}}$. This transfer operator is $\left(\ell^{p}, \ell^{p}\right)$-stable for $p=0,1, \cdots, \infty$, i.e., maps vectors with components in $\ell^{p}$ onto vectors with the same properties. This is well known from the LTI case.

The standard action

$$
\circ: \mathbf{A} \times W \rightarrow W \text { with }(q \circ w)(t):=w(t+1),(a \circ w)(t):=a(t) w(t),
$$

for $a \in \mathbb{F}^{\mathbb{Z} / \mathbb{Z} N}, w \in W, t \in \mathbb{N}$ makes $W$ an injective A-left module, but not a cogenerator, cf. Thm. 3.4 and Remark 3.5. As usual this action is extended to one of a matrix

$$
\begin{gathered}
R=\sum_{j=0}^{d} R_{j} q^{j} \in \mathbf{A}^{r \times k}, R_{j} \in\left(\mathbb{F}^{\mathbb{Z} / \mathbb{Z} N}\right)^{r \times k} \text { on } w=\left(w_{1}, \cdots, w_{k}\right)^{\top} \in W^{k}:=W^{k \times 1}: \\
(R \circ w)(t)=\sum_{j=0}^{d} R_{j}(t) w(t+j), \mathcal{B}:=\left\{w \in W^{k} ; R \circ w=0\right\} .
\end{gathered}
$$

The equation $R \circ w=0$ is a linear system of difference equations with $N$-periodic

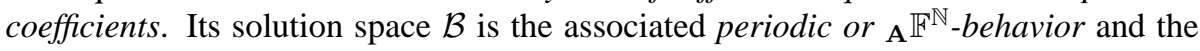
principal object of study in this paper. 
The center of $\mathbf{A}$ is the commutative polynomial algebra $\mathbf{Z}:=\mathbb{F}[\Delta], \Delta:=q^{N}$, that acts on the signal space $\widehat{W}:=\mathbb{F}^{\mathbb{N} N}$ via left shift, i.e.,

$$
(\Delta \circ \widehat{w})(\tau):=\widehat{w}(\tau+N), \widehat{w} \in \widehat{W}=\mathbb{F}^{\mathbb{N} N}, \tau \in \mathbb{N} N=\{0, N, 2 N, \cdots\},
$$

and makes it an injective cogenerator with its ensuing categorical duality between LTI $\mathbf{z} \widehat{W}$-behaviors and finitely generated (f.g.) Z-modules, cf. [5, (20)-(22)]. By means of the isomorphism

$$
\begin{gathered}
W=\mathbb{F}^{\mathbb{N}} \cong \widehat{W}^{N}=\left(\mathbb{F}^{\mathbb{N} N}\right)^{N}, w \mapsto \widehat{w}:=\left(w_{0}, \cdots w_{N-1}\right)^{\top} \text { with } \\
w(j+\tau):=w_{j}(\tau), 0 \leq j \leq N-1, \tau \in \mathbb{N} N
\end{gathered}
$$

we derive a categorical equivalence between periodic behaviors, i.e., $\mathbf{A} W$-behaviors, and $\mathbf{z} \widehat{W}$-behaviors (Thm. 3.4) that is our formulation of the correspondence of periodic behaviors and their lifted LTI form, cf. [5, Thm. 4.6]. It enables the transfer of Vidyasagar's LTI stabilization and control theory [16] to periodic behaviors and the application of [3]. The algebra $\mathbf{A}$ is canonically a subalgebra of the matrix algebra $\mathbf{B}:=\mathbf{Z}^{N \times N}$. Since $\mathbf{A} W$ is not a cogenerator, periodic behaviors are not dual to f.g. A-left modules, but to f.g. B-left modules (Thm. 3.14). This is shown by means of the isomorphism (7) and by Morita's theory of equivalent module categories that also implies the precise structure of these modules. F.g. A-modules have a more complicated structure and were studied in [9], but are not employed for the study of periodic behaviors in the present paper.

The main results of this paper described above are contained in Sections 5 and 6. Section 3 describes the module-behavior duality for periodic behaviors on the time-axis $\mathbb{N}$. For the time-axis $\mathbb{Z}$ the theory is simpler and was treated with similar methods in [5] where we also explained the relation with previous work [11], [1], [2]. In Section 4 we apply Morita theory to derive essential notions for and properties of periodic behaviors and their dual f.g. B-left modules, for instance autonomy, controllability, the existence and characterization of input/output (IO) structures and left and right coprime factorizations. We show that in the Morita framework a periodic behavior and its lifted LTI behavior coincide via the isomorphism (7). This simplifies the considerations of Sections 5 and 6 considerably. In Section 5 we also discuss the characteristic variety or set of poles of an autonomous behavior and define and characterize the stability of autonomous and of input/output systems.

By means of the algorithms from [3] all results of this paper are constructive, but have not yet been implemented in the periodic case.

History: A completely different approach to stabilization and control of discrete periodic systems given by state space equations is exposed by Bittanti and Colaneri [2, pp. 353-404], see also [8] and [17] (continuous time). Commutative and noncommutative rings of (partial) differential operators and their modules have been an important tool in Algebraic Analysis since the seminal work of Ehrenpreis, Malgrange and Palamodov for constant coefficients in the 1960s and later, for varying coefficients, in the work of Kashiwara and many other researchers. In systems theory already Kalman employed polynomial modules, but from a different point of view, and Ylinen [18] already used skew-polynomial rings of differential operators. In connection with Rosenbrock's polynomial and Willems' behavioral approach modules were introduced by Fliess and the second author in 1990, also for multidimensional behaviors, and were also used in [9]. The module theoretic reformulation of the fractional representation approach (cf. the bibliographies of [16] and [14] for important contributors to the latter field) and its 
application to stabilization problems is due to Quadrat, cf. [14], and was also applied in [3]. In this approach a commutative domain $\mathbf{S}$ of stable operators, often a Banach algebra, with its quotient field $\mathbf{K}$ is given. A system is described by a transfer matrix $H \in \mathbf{K}^{r \times k}$, hence hidden modes are removed and autonomous systems as in Section 6 cannot be used. A similar framework is used in [6]. Our ring $\mathbf{B}$ of difference operators is neither commutative nor a domain, but the Morita theory enables the reduction of the problems to the commutative operator domains $\mathbb{F}[s]$ and $\mathcal{S}$, as used in [16] and [3].

\section{Terminology and notations}

We have to use various notions from algebra. We refer to the books [15], [13] and [12] for the basic algebraic language concerning rings, modules and categories. For the convenience of the reader we give here a list of notations with short explanations, essentially in the order in which they appear in the paper:

1. Abbreviations: f.d.=finite-dimensional, f.g.=finitely generated, $\mathrm{IO}=$ input/output, LTI=linear time-invariant, LTV=linear time-varying, resp.=respectively, w.l.o.g.= without loss of generality, w.r.t.=with respect to

2. $X^{r \times k}:=$ the abelian group of $r \times k$-matrices with entries in the abelian group $X, X^{1 \times k}:=$ rows, $X^{k}:=X^{k \times 1}:=$ columns

3. $\mathbb{F}=$ a field, $\mathbb{F}=\mathbb{R}, \mathbb{C}$ in Sections 5 and 6

4. $\mathbb{F}^{\mathbb{Z} / \mathbb{Z} N}\left(\underset{\text { identification }}{\subset} \mathbb{F}^{\mathbb{N}}\right)$ : the commutative coefficient ring of periodic functions $a: \mathbb{N} \rightarrow \mathbb{F}$ of period $N$ with $a(\bar{t}):=a(t), \bar{t}:=t+\mathbb{Z} N \in \mathbb{Z} / \mathbb{Z} N, t \in \mathbb{N}$

5. $\epsilon_{\bar{i}}=\epsilon_{\bar{i}}^{2}, \epsilon_{\bar{i}}(\bar{j})=\delta_{\bar{i}, \bar{j}}, \bar{i}, \bar{j} \in \mathbb{Z} / \mathbb{Z} N$ : the standard $\mathbb{F}$-basis of $\mathbb{F}^{\mathbb{Z} / \mathbb{Z} N}$ consisting of idempotents

6. $\mathbf{A}:=\mathbb{F}^{\mathbb{Z} / \mathbb{Z} N}[q ; \circ]$ : the noncommutative $\mathbb{F}$-algebra of skew-polynomials in the indeterminate $q$ with coefficients in $\mathbb{F}^{\mathbb{Z} / \mathbb{Z} N}$

7. $\mathbf{Z}:=\operatorname{center}(\mathbf{A}):=\{z \in \mathbf{A} ; \forall a \in \mathbf{A}: a z=z a\}=\mathbb{F}[\Delta]:$ the center of $\mathbf{A}$ and polynomial algebra in the indeterminate $\Delta:=q^{N}$ with coefficients in $\mathbb{F}$

8. $W=\mathbb{F}^{\mathbb{N}}:=$ the space of signal functions (sequences) $w: \mathbb{N} \rightarrow \mathbb{F}$ and A-left module with the shift action $(q \circ w)(t):=w(t+1)$ and $(a \circ w)(t):=a(t) w(t)$ for $a \in \mathbb{F}^{\mathbb{Z} / \mathbb{Z} N}$

9. $\widehat{W}:=\mathbb{F}^{\mathbb{N} N}:=$ the space of signals (sequences) $\widehat{w}: \mathbb{N} N \rightarrow \mathbb{F}$ and Z-module with the action $(\Delta \circ \widehat{w})(j N)=\widehat{w}(j N+N)=\widehat{w}((j+1) N), W \cong \widehat{W}^{N}$

10. $\mathbf{A}$ Mod $:=$ the class or category of A-left modules

11. $\operatorname{Hom}_{\mathbf{A}}(M, N)$ : the $\mathbf{Z}$-module of $\mathbf{A}$-linear maps between A-left modules $M, N$

12. $\mathcal{Z}: \mathbf{A} \operatorname{Mod} \rightarrow_{\mathbf{Z}} \operatorname{Mod}, M \mapsto \epsilon_{\overline{0}} M$ : the exact left adjoint to $\mathcal{A}$

13. $\mathcal{A}: \mathbf{z} \operatorname{Mod} \rightarrow_{\mathbf{A}} \operatorname{Mod}, P \mapsto P^{N}$ : the exact right adjoint to $\mathcal{Z}$

14. $\mathbf{B}=\mathbf{Z}^{N \times N} \underset{\text { identification }}{\supset} \mathbf{A}: \mathbf{Z}$-algebra of $N \times N$-matrices

15. $\mathbb{S}_{\mathbf{Z}}:=\mathbf{Z} \backslash\{0\}$ : multiplicative monoid of nonzero polynomials in $\mathbf{Z}=\mathbb{F}[\Delta]$ 
16. $\mathbf{K}:=\mathbf{Z}_{\mathbb{S}_{\mathbf{Z}}}=\mathbb{F}(\Delta)$ : quotient ring of $\mathbf{Z}$ w.r.t. $\mathbb{S}_{\mathbf{Z}}$, quotient field of $\mathbf{Z}$

17. $\mathbf{Q}:=\mathbf{B}_{\mathbb{S}_{\mathbf{Z}}}=\mathbf{K}^{N \times N}$ : quotient ring of $\mathbf{B}$ with denominators in $\mathbb{S}_{\mathbf{Z}}$ and matrix ring over the field $\mathbf{K}$

18. $\mathbb{F}(\Delta)_{\operatorname{pr}} \subset \mathbb{F}(\Delta)$ : ring of proper rational functions in $\Delta$

19. In the following: $\mathbb{F}=\mathbb{R}, \mathbb{C}$

20. $\mathbb{D} \subseteq\{\lambda \in \mathbb{C} ;|\lambda|<1\}$ : nonempty (open) subset of the open unit disc

21. $\mathbb{S}_{\mathbb{D}} \subset \mathbb{S}_{\mathbf{Z}}$ : saturated monoid of $(\mathbb{D})$-stable polynomials, i.e., with roots in $\mathbb{D}$

22. $\mathbf{Z}_{\mathbb{D}}:=\mathbf{Z}_{\mathbb{S}}$ : quotient ring of (D)-stable rational functions with denominators in $\mathbb{S}_{\mathbb{D}}$

23. $\mathbf{B}_{\mathbb{D}}:=\mathbf{B}_{\mathbb{S}_{\mathbb{D}}}, M_{\mathbb{D}}:=M_{\mathbb{S}_{\mathbb{D}}}$ : quotient ring and module

24. $W_{\mathbb{D}}$, resp. $\widehat{W}_{\mathbb{D}}$ : injective cogenerator quotient signal modules over $\mathbf{B}_{\mathbb{D}}$, resp. $\mathbf{Z}_{\mathbb{D}}$

25. $\mathbf{S}:=\mathbf{Z}_{\mathbb{D}} \cap \mathbb{F}(\Delta)_{\text {pr }}$ : ring of proper and $(\mathbb{D})$-stable rational functions

26. $\mathbf{C}:=\mathbf{S}^{N \times N}$ : matrix ring over $\mathbf{S}$

27. $\mathcal{B} \subseteq W^{p+m}$ : input/output ${ }_{\mathrm{B}} W$-behavior, $\mathcal{B}^{0}$ : its autonomous part, $\mathcal{B}_{\mathbb{D}} \subseteq W_{\mathbb{D}}^{p+m}$ : its quotient

28. $\widehat{\mathcal{B}} \subseteq \widehat{W}^{\ell}$ : autonomous $\mathbf{z} \widehat{W}$-behavior, $\operatorname{char}(\widehat{\mathcal{B}})$ : its characteristic variety or set of characteristic values or poles

\section{Module-behavior duality for periodic behaviors on $\mathbb{N}$}

We treat discrete periodic behaviors on the time-axis $\mathbb{N}$ in analogy to the case of the lattice $\mathbb{Z}^{r}[5$, \$4]. The main goal is the proof of Thm. 3.14 that describes the equivalence between periodic behaviors and their lifted LTI forms and the duality of these to their associated modules constructively. Morita equivalence plays a decisive part.

Consider the cyclic group $\mathbb{Z} / \mathbb{Z} N, N>0$, with the elements $\bar{i}:=i+\mathbb{Z} N, i \in \mathbb{Z}$, a field $\mathbb{F}$, the time axis $\mathbb{N}$ and the signal space $W:=\mathbb{F}^{\mathbb{N}}$. The algebra $\mathbb{F}^{\mathbb{Z} / \mathbb{Z} N}$ with the componentwise multiplication is identified with the subalgebra of $N$-periodic functions on $\mathbb{N}$, i.e.,

$$
\mathbb{F}^{\mathbb{Z} / \mathbb{Z} N}=\left\{a \in \mathbb{F}^{\mathbb{N}} ; \forall t \in \mathbb{N}: a(t)=a(t+N)\right\}, a(\bar{t})=a(t) .
$$

It has the $\mathbb{F}$-basis $\epsilon_{\bar{i}}, \bar{i} \in \mathbb{Z} / \mathbb{Z} N$, of complete orthogonal idempotents defined by $\epsilon_{\bar{i}}(t)=\delta_{\bar{i}, \bar{t}}$. As in [5] the monoid $\mathbb{N}$ acts on $\mathbb{Z} / \mathbb{Z} N$, resp. on $\mathbb{F}^{\mathbb{Z} / \mathbb{Z} N}$ by $i \circ \bar{j}=\bar{i}+\bar{j}$, resp. by $(i \circ a)(\bar{t})=a(\bar{i}+\bar{t})$ and then

$$
\mathbb{F}^{\mathbb{Z} / \mathbb{Z} N}=\oplus_{\bar{i} \in \mathbb{Z} / \mathbb{Z} N} \mathbb{F} \epsilon_{\bar{i}}=\oplus_{i=0}^{N-1} \mathbb{F} \epsilon_{\bar{i}}, j \circ \epsilon_{\bar{i}}=\epsilon_{\bar{i}-\bar{j}} .
$$


As in [5, (23)-(25), (69)-(74)] we get the (noncommutative) skew-polynomial algebra (cf. (1))

$$
\begin{aligned}
& \mathbf{A}:=\mathbb{F}^{\mathbb{Z} / \mathbb{Z} N}[q ; \circ]=\oplus_{\bar{i} \in \mathbb{Z} / \mathbb{Z} N, j \in \mathbb{N}} \mathbb{F} \epsilon_{\bar{i}} q^{j}, q^{j} \epsilon_{\bar{i}}=\epsilon_{\bar{i}-\bar{j}} q^{j} \\
& \mathbf{Z}:=\operatorname{center}(\mathbf{A})=\mathbb{F}[\Delta], \Delta:=q^{N}, \\
& \mathbf{A}=\oplus_{i, j=0}^{N-1} \mathbf{Z} \epsilon_{\bar{i}} q^{j}, \epsilon_{\bar{i}} \mathbf{A}=\oplus_{j=0}^{N-1} \mathbf{Z} \epsilon_{i} q^{j}, \mathbf{A} \epsilon_{\bar{i}}=\oplus_{j=0}^{N-1} \mathbf{Z} \epsilon_{\bar{i}-\bar{j}} q^{j}, \\
& \epsilon_{\overline{0}} \mathbf{A}=\oplus_{j=0}^{N-1} \mathbf{Z} \epsilon_{\overline{0}} q^{j}, \epsilon_{\overline{0}} \mathbf{A} \epsilon_{\overline{0}}=\mathbf{Z} \epsilon_{\overline{0}} \\
& \mathbf{v}:=\left(\begin{array}{c}
\epsilon_{\overline{0}} \\
\cdots \\
\epsilon_{\overline{0}} q^{N-1}
\end{array}\right) \in\left(\epsilon_{\overline{0}} \mathbf{A}\right)^{N}, \epsilon_{\overline{0}} \mathbf{A}=\mathbf{Z}^{1 \times N} \mathbf{v} .
\end{aligned}
$$

The algebra $\mathbf{A}$ acts on the signal space $W=\mathbb{F}^{\mathbb{N}}$ by means of (4) and makes it an A-left module. A matrix $R \in \mathbf{A}^{r \times k}$ gives rise to the equation module $U:=$ $\mathbf{A}^{1 \times r} R \subseteq \mathbf{A}^{1 \times k}$, the system factor module $M:=\mathbf{A}^{1 \times k} / U$ and the behavior $\mathcal{B}:=$ $\left\{w \in W^{\bar{k}} ; R \circ w=0\right\}$. The following simple, but important $\mathbb{F}$-linear isomorphism

$$
\begin{gathered}
\operatorname{Hom}_{\mathbf{A}}(M, W) \underset{\text { Malgrange } 1962}{\cong} \mathcal{B}, \Phi \mapsto w=\left(w_{1}, \cdots, w_{k}\right)^{\top}, \\
W=\mathbb{F}^{\mathbb{N}}, \Phi(\xi+U)=\xi w=\sum_{j=1}^{k} \xi_{j} \circ w_{j}, \xi=\left(\xi_{1}, \cdots, \xi_{k}\right) \in \mathbf{A}^{1 \times k},
\end{gathered}
$$

holds and shows that the ubiquitous Hom-spaces (see Thm. 3.4 below)

$$
\operatorname{Hom}_{\mathbf{A}}(M, W) \cong \operatorname{Hom}_{\mathbf{Z}}\left(\epsilon_{\overline{0}} M, \widehat{W}\right), \widehat{W}=\mathbb{F}^{\mathbb{N} N},
$$

and the results about them have a direct systems theoretic significance. For f.g. Aleft modules $M$ with a given representation $M=\mathbf{A}^{1 \times k} / U$ as in (11) the Malgrange isomorphism is canonical (functorial) and hence we identify $\mathcal{B}=\operatorname{Hom}_{\mathbf{A}}(M, W)$.

Since $\epsilon_{\bar{i}}$ is idempotent $\mathbf{A} \epsilon_{\bar{i}}$ is a projective direct summand of $\mathbf{A}$, but, in contrast to the case of the time-axis $\mathbb{Z}[5]$, the $\mathbf{A} \epsilon_{\bar{i}}$ are not isomorphic to $\mathbf{A} \epsilon_{\overline{0}}$ and the latter is not a progenerator, i.e., a f.g. projective generator of ${ }_{\mathbf{A}} \mathbf{M o d}$. The module $\epsilon_{\overline{0}} \mathbf{A}$ is a $(\mathbf{Z}, \mathbf{A})$ - bimodule and free of dimension $N$ as $\mathbf{Z}$-module with the $\mathbf{Z}$-basis $\mathbf{v}$. We identify $\mathbf{Z}^{1 \times N} \underset{\text { ident. }}{=} \epsilon_{\overline{0}} \mathbf{A}$ by $z=\left(z_{0}, \cdots, z_{N-1}\right) \underset{\text { ident. }}{=} z \mathbf{v}$. If $P$ is any $\mathbf{Z}$-module then $\operatorname{Hom}_{\mathbf{Z}}\left(\epsilon_{\overline{0}} \mathbf{A}, P\right)$ is a left $\mathbf{A}$-module with the action

$$
(a \varphi)\left(\epsilon_{\overline{0}} b\right)=\varphi\left(\epsilon_{\overline{0}} b a\right), a, b \in \mathbf{A}, \varphi \in \operatorname{Hom}_{\mathbf{Z}}\left(\epsilon_{\overline{0}} \mathbf{A}, P\right) .
$$

The map

$$
\operatorname{Hom}_{\mathbf{Z}}\left(\epsilon_{\overline{0}} \mathbf{A}, P\right) \cong P^{N}:=P^{N \times 1}, \varphi \mapsto \varphi(\mathbf{v})=: y=\left(y_{0}, \cdots, y_{N-1}\right)^{\top}, y_{j}=\varphi\left(\epsilon_{0} q^{j}\right),
$$

is a Z-isomorphism. We identify $\operatorname{Hom}_{\mathbf{Z}}\left(\epsilon_{\overline{0}} \mathbf{A}, P\right)=P^{N}, \varphi=\varphi(\mathbf{v})$. We turn $P^{N}$ into an A-left module by transport of structure along the isomorphism of (14), hence

$$
\begin{aligned}
& a y=a \varphi(\mathbf{v}):=\varphi(\mathbf{v} a) \\
& \epsilon_{\bar{j}} y=\left(0, \cdots, 0, \stackrel{j}{y}_{j}, 0, \cdots, 0\right)^{\top}, j=0, \cdots, N-1 \\
& q y=\left(y_{1}, \cdots, y_{N-1}, \Delta \circ y_{0}\right)^{\top}, \Delta=q^{N} \in \mathbf{Z} .
\end{aligned}
$$


Corollary 3.1. Consider the signal modules $\mathbf{A} \mathbb{F}^{\mathbb{N}}$ and $\mathbf{Z} \mathbb{F}^{\mathbb{N} N}$. There is the $\mathbf{Z}$-isomorphism

$$
\begin{gathered}
\mathbb{F}^{\mathbb{N}} \cong \operatorname{Hom}_{\mathbf{Z}}\left(\epsilon_{\overline{0}} \mathbf{A}, \mathbb{F}^{\mathbb{N} N}\right) \cong\left(\mathbb{F}^{\mathbb{N} N}\right)^{N}, w \mapsto \widehat{w}=\left(\begin{array}{c}
w_{0} \\
w_{N-1}
\end{array}\right), \text { with } \\
w_{i}(\tau):=w(i+\tau), 0 \leq i \leq N-1, \tau \in \mathbb{N} N .
\end{gathered}
$$

This isomorphism is even A-linear where $\left(\mathbb{F}^{\mathbb{N} N}\right)^{N} \cong \operatorname{Hom}_{\mathbf{Z}}\left(\epsilon_{\overline{0}} \mathbf{A}, \mathbb{F}^{\mathbb{N} N}\right)$ has the $\mathbf{A}$ structure from (15).

We define the two functors

$$
\begin{aligned}
& \mathcal{Z}:{ }_{\mathbf{A}} \operatorname{Mod} \rightarrow{ }_{\mathbf{z}} \text { Mod, } M \mapsto \mathcal{Z}(M):=\epsilon_{\overline{0}} M, \text { and } \\
& \mathcal{A}:{ }_{\mathbf{z}} \operatorname{Mod} \rightarrow{ }_{\mathbf{A}} \text { Mod, } P \mapsto \mathcal{A}(P):=\operatorname{Hom}_{\mathbf{Z}}\left(\epsilon_{\overline{0}} \mathbf{A}, P\right)=P^{N} .
\end{aligned}
$$

The functors are exact since $\mathbf{A} \epsilon_{\overline{0}}$, resp. $\epsilon_{\overline{0}} \mathbf{A}$ are $\mathbf{A}$-projective, resp. $\mathbf{Z}$-free.

Corollary 3.2. ([9, Thm. 5]) The module $\mathbf{A} \epsilon_{\overline{0}}$ is f.g., projective as direct summand of A, but not free.

Proof. Assume

$$
\mathbf{A} \epsilon_{\overline{0}} \underset{\mathbf{A}}{\cong} \mathbf{A}^{1 \times m} \Longrightarrow \mathbf{Z} \epsilon_{\overline{0}}=\epsilon_{\overline{0}} \mathbf{A} \epsilon_{\overline{0}} \cong\left(\epsilon_{\overline{0}} \mathbf{A}\right)^{1 \times m} \Longrightarrow 1=\operatorname{dim} \mathbf{Z}\left(\left(\epsilon_{\overline{0}} \mathbf{A}\right)^{1 \times m}\right)=m N .
$$

This is a contradiction.

Lemma 3.3. For $M \in{ }_{\mathrm{A}} \mathrm{Mod}$ and $P \in{ }_{\mathrm{z}} \mathrm{Mod}$ there is the functorial isomorphism

$$
\begin{gathered}
\operatorname{Hom}_{\mathbf{Z}}\left(\epsilon_{\overline{0}} M, P\right) \cong \operatorname{Hom}_{\mathbf{A}}\left(M, P^{N}\right), \varphi \mapsto \Phi, \text { where } \\
\Phi(x)=\left(\varphi\left(\epsilon_{\overline{0}} x\right), \cdots, \varphi\left(\epsilon_{\overline{0}} q^{N-1} x\right)\right)^{\top}, \varphi\left(\epsilon_{\overline{0}} x\right)=\Phi(x)_{0} .
\end{gathered}
$$

The isomorphism means that $\mathcal{Z}(\mathcal{A})$ is left (right) adjoint to $\mathcal{A}(\mathcal{Z})$ [15, \$IV.9]

Proof. The isomorphism follows from $\varphi\left(\epsilon_{\overline{0}} q^{j} x\right)=\Phi\left(q^{j} x\right)_{0}=\left(q^{j} \Phi(x)\right)_{0}=\Phi(x)_{j}$.

We recall that an A-module ${ }_{\mathbf{A}} W$ is called injective if the contravariant functor

$$
\operatorname{Hom}_{\mathbf{A}}(-, W):{ }_{\mathbf{A}} \operatorname{Mod} \rightarrow{ }_{\mathbf{z}} \operatorname{Mod}, M \mapsto \operatorname{Hom}_{\mathbf{A}}(M, W),
$$

preserves the exactness of sequences or, equivalently, maps monomorphisms to epimorphisms. If $\mathbf{A}_{\mathbf{A}} W$ is injective it is also a cogenerator if and only if $\operatorname{Hom}_{\mathbf{A}}(M, W)=0$ implies $M=0$.

Theorem 3.4. The isomorphisms (16) and (19) imply the functorial isomorphism

$$
\begin{aligned}
& \widehat{\mathcal{B}}:=\operatorname{Hom}_{\mathbf{Z}}\left(\epsilon_{\overline{0}} M, \mathbb{F}^{\mathbb{N} N}\right) \cong \mathcal{B}:=\operatorname{Hom}_{\mathbf{A}}\left(M, \mathbb{F}^{\mathbb{N}}\right), \varphi \leftrightarrow \Phi, \text { where } \\
& \Phi(x)(j+\tau)=\varphi\left(\epsilon_{\overline{0}} q^{j} x\right)(\tau), x \in M, j=0, \cdots, N-1, \tau \in \mathbb{N} N .
\end{aligned}
$$

Since $\mathcal{Z}: M \mapsto \epsilon_{\overline{0}} M$ is exact and since $\mathbf{Z}^{\mathbb{N} N}$ is the standard LTI injective cogenerator, the signal module $\mathbf{A} \mathbb{F}^{\mathbb{N}}$ is injective too and

$$
\epsilon_{\overline{0}} M=0 \Longleftrightarrow \operatorname{Hom}_{\mathbf{A}}\left(M, \mathbb{F}^{\mathbb{N}}\right)=0 .
$$

Hence any (periodic) ${ }_{\mathbf{A}} \mathbb{F}^{\mathbb{N}}$-behavior $\mathcal{B}$ is canonically an $L T I \mathbb{Z}^{\mathbb{N} N}$-behavior $\widehat{\mathcal{B}}$. 
Since $M \mapsto \epsilon_{\overline{0}} M$ and $M \mapsto \operatorname{Hom}_{\mathbf{A}}\left(M, \mathbb{F}^{\mathbb{N}}\right)$ are exact the full subcategory

$$
\mathfrak{C}:=\left\{C \in{ }_{\mathbf{A}} \operatorname{Mod} ; \epsilon_{\overline{0}} C=0 \text { or } \operatorname{Hom}_{\mathbf{A}}\left(C, \mathbb{F}^{\mathbb{N}}\right)=0\right\}
$$

is a Serre subcategory, i.e., closed under isomorphisms, submodules, factor modules, extensions and direct sums.

Remark 3.5. The category $\mathfrak{C}$ contains nonzero modules, and hence ${ }_{\mathbf{A}} \mathbb{F}^{\mathbb{N}}$ is not a cogenerator.

The largest submodule of $M$ in $\mathfrak{C}$ is called its ( $\mathfrak{C}$ )-radical and denoted by

$$
\operatorname{Ra}(M)=\left\{x \in M ; \epsilon_{\overline{0}} \mathbf{A} x=0\right\} .
$$

The representation $\epsilon_{\overline{0}} \mathbf{A}=\oplus_{j=0}^{N-1} \mathbf{Z} \epsilon_{\overline{0}} q^{j}$ and a simple computation imply

$$
\begin{aligned}
\operatorname{Ra}(\mathbf{A}) & =\left\{a \in \mathbf{A} ; \epsilon_{\overline{0}} \mathbf{A} a=0\right\} \\
& =\left\{a \in \mathbf{A} ; \forall j=0, \cdots, N-1: \epsilon_{\overline{0}} q^{j} a=0\right\}=0 .
\end{aligned}
$$

As usual the adjointness implies the functorial morphisms [15, Prop. 9.3]

$$
\begin{gathered}
\zeta: \mathcal{Z A} \rightarrow \operatorname{id}_{\mathbf{z M o d}}, \eta: \operatorname{id}_{\mathbf{A} \text { Mod }} \rightarrow \mathcal{A Z} \\
\zeta_{P}: \epsilon_{\overline{0}} P^{N} \rightarrow P, \epsilon_{\overline{0}}\left(y_{0}, \cdots, y_{N-1}\right)^{\top}=\left(y_{0}, 0, \cdots, 0\right)^{\top} \mapsto y_{0} \\
\eta_{M}: M \rightarrow \epsilon_{\overline{0}} M^{N}, x \mapsto\left(\epsilon_{\overline{0}} q^{0} x, \cdots, \epsilon_{\overline{0}} q^{N-1} x\right)^{\top} .
\end{gathered}
$$

The morphism $\zeta$ is obviously an isomorphism. Like all adjointness morphisms these satisfy the relations

$$
\begin{aligned}
& \mathcal{A} \stackrel{\eta_{\mathcal{A}}}{\longrightarrow} \mathcal{A Z} \mathcal{A} \stackrel{\mathcal{A} \zeta}{\longrightarrow} \mathcal{A}, \mathcal{A}\left(\zeta_{P}\right) \eta_{\mathcal{A}(P)}=\operatorname{id}_{\mathcal{A}(P)} \\
& \mathcal{Z} \stackrel{\mathcal{Z} \eta}{\longrightarrow} \mathcal{Z} \mathcal{A} \stackrel{\zeta_{\mathcal{Z}}}{\longrightarrow} \mathcal{Z}, \zeta_{\mathcal{Z}(M)} \mathcal{Z}\left(\eta_{M}\right)=\operatorname{id}_{\mathcal{Z}(M)} .
\end{aligned}
$$

Corollary 3.6. For every $P$ the map $\eta_{\mathcal{A}(P)}: \mathcal{A}(P) \rightarrow \mathcal{A Z} \mathcal{A}(P)$ is an isomorphism.

Proof. This follows from $\mathcal{A}\left(\zeta_{P}\right) \eta_{\mathcal{A}(P)}=\operatorname{id}_{\mathcal{A}(P)}$ and the isomorphy of $\zeta_{P}$.

An A-module $M$ is called closed if $\eta_{M}$ is an isomorphism. Let $\left(\mathbf{A}, \epsilon_{\overline{0}}\right)$ Mod denote the full subcategory of ${ }_{\mathbf{A}}$ Mod of all closed A-modules. The adjointness of (19) and Cor. 3.6 imply

Corollary 3.7. (cf. [15, Prop. XI.8.7]) The adjoint functors $\mathcal{Z}$ and $\mathcal{A}$ imply the inverse categorical equivalences

$$
\begin{aligned}
& \mathcal{Z}:{ }_{\left(\mathbf{A}, \epsilon_{\overline{0}}\right)} \operatorname{Mod} \stackrel{\cong}{\mathbf{z}} \text { Mod, } M \mapsto \mathcal{Z} M=\epsilon_{\overline{0}} M,
\end{aligned}
$$

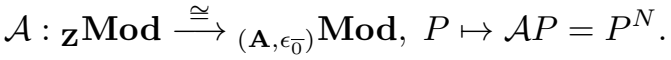

Since $\mathcal{Z}$ and $\mathcal{A}:{ }_{\mathbf{z}}$ Mod $\rightarrow{ }_{\mathbf{A}}$ Mod are exact the subcategory ${ }_{\left(\mathbf{A}, \epsilon_{\overline{0}}\right)}$ Mod of closed modules is closed under isomorphisms, kernels, cokernels and extensions and, in particular, abelian.

In slightly superficial terms a categorical equivalence between categories is a oneone correspondence between the classes of objects with natural (functorial) properties. 
Corollary 3.8. For all $M \in{ }_{\mathbf{A}} \operatorname{Mod}$ and $C \in{ }_{\left(\mathbf{A}, \epsilon_{\overline{0}}\right)}$ Mod the isomorphism

$$
\operatorname{Hom}_{\mathbf{A}}(\mathcal{A Z M}, C) \cong \operatorname{Hom}_{\mathbf{A}}(M, C), \Phi \mapsto \Phi \eta_{M},
$$

holds. This means that the exact functor $M \mapsto \mathcal{A Z} M=\epsilon_{\overline{0}} M^{N}$ is left adjoint to the inclusion $\left(\mathbf{A}, \epsilon_{\overline{0}}\right)$ Mod $\subset{ }_{\mathbf{A}}$ Mod.

Proof. This follows from the commutative diagram

$$
\begin{array}{ccc}
M & \stackrel{\eta_{M}}{\longrightarrow} & \mathcal{A Z M}=\epsilon_{\overline{0}} M^{N} \\
\downarrow \varphi & & \downarrow \mathcal{A Z} \varphi \\
C & \stackrel{\eta_{C} \cong}{\longrightarrow} & \mathcal{A Z C}
\end{array} \Longrightarrow \varphi=\left(\eta_{C}^{-1} \mathcal{A Z} \varphi\right) \eta_{M} .
$$

Theorem 3.9. According to Cor. $3.1 \eta_{\mathbb{F}^{\mathbb{N}}}$ is an isomorphism. Hence ${ }_{\mathbf{A}} \mathbb{F}^{\mathbb{N}}$ is closed and indeed an injective cogenerator in the category ${ }_{\left(\mathbf{A}, \epsilon_{\overline{0}}\right)}$ Mod of closed $\mathbf{A}$-modules. For all $M \in{ }_{\mathbf{A}}$ Mod the map

$$
\operatorname{Hom}_{\mathbf{A}}\left(\mathcal{A Z} M, \mathbb{F}^{\mathbb{N}}\right) \cong \operatorname{Hom}_{\mathbf{A}}\left(M, \mathbb{F}^{\mathbb{N}}\right), \Phi \mapsto \Phi \eta_{M},
$$

is an isomorphism according to (29). Thus every $\mathbf{A}^{\mathbb{N}} \mathbb{F}^{\mathbb{N}}$-behavior is described by a unique closed module. The functor

$$
\left(\mathbf{A}, \epsilon_{\bar{\sigma}}\right) \operatorname{Mod}^{\mathrm{fg}} \rightarrow\left\{\mathbf{A} \mathbb{F}^{\mathbb{N}} \text {-behaviors }\right\}, M \mapsto \operatorname{Hom}_{\mathbf{A}}\left(M, \mathbb{F}^{\mathbb{N}}\right),
$$

thus establishes a categorical duality between the category of f.g. closed A-left modules and that of (periodic) $\mathbf{A}^{\mathbb{N}}$-behaviors.

Lemma 3.10. For all $M$ the kernel and cokernel of $\eta_{M}$ belong to $\mathfrak{C}$, more precisely

$$
\operatorname{ker}\left(\eta_{M}\right)=\operatorname{Ra}(M), \epsilon_{\overline{0}} \operatorname{ker}\left(\eta_{M}\right)=0 \text { and } \epsilon_{\overline{0}} \operatorname{cok}\left(\eta_{M}\right)=0 .
$$

Proof. (i) We apply the exact functor $\mathcal{Z}: M \mapsto \epsilon_{\overline{0}} M$ to the exact sequence

$$
\begin{aligned}
& 0 \rightarrow \operatorname{ker}\left(\eta_{M}\right) \stackrel{\text { inj }}{\longrightarrow} M \stackrel{\eta_{M}}{\longrightarrow} \mathcal{A Z} M \stackrel{\text { can }}{\longrightarrow} \operatorname{cok}\left(\eta_{M}\right) \rightarrow 0, \\
& \text { hence the sequence } \\
& 0 \rightarrow \mathcal{Z} \operatorname{ker}\left(\eta_{M}\right) \stackrel{\mathcal{Z} \text { inj }}{\longrightarrow} \mathcal{Z} M \stackrel{\mathcal{Z} \eta_{M}}{\longrightarrow} \mathcal{Z} \mathcal{A Z} M \stackrel{\mathcal{Z} \operatorname{can}}{\longrightarrow} \mathcal{Z} \operatorname{cok}\left(\mathcal{Z} \eta_{M}\right) \rightarrow 0
\end{aligned}
$$

is also exact. But $\zeta_{\mathcal{Z}(M)} \mathcal{Z}\left(\eta_{M}\right)=\operatorname{id}_{\mathcal{Z}(M)}$ by (27) and $\zeta$ is an isomorphism, hence also $\mathcal{Z} \eta_{M}=\zeta_{\mathcal{Z}(M)}^{-1}$ is an isomorphism. This implies $\mathcal{Z} \operatorname{ker}\left(\eta_{M}\right)=\epsilon_{0} \operatorname{ker}\left(\eta_{M}\right)=0$ and likewise $\mathcal{Z} \operatorname{cok}\left(\eta_{M}\right)=\epsilon_{\overline{0}} \operatorname{cok}\left(\eta_{M}\right)=0$.

(ii) The equation $\epsilon_{0} \operatorname{ker}\left(\eta_{M}\right)=0$ implies $\operatorname{ker}\left(\eta_{M}\right) \subseteq \operatorname{Ra}(M)$. Conversely, $\epsilon_{\overline{0}} \operatorname{Ra}(M)=$ 0 implies the commutative exact diagram

$$
\begin{aligned}
& 0 \rightarrow \operatorname{Ra}(M)) \quad \stackrel{\text { inj }}{\longrightarrow} \quad M \quad \stackrel{\text { can }}{\longrightarrow} M / \operatorname{Ra}(M) \quad \rightarrow 0
\end{aligned}
$$

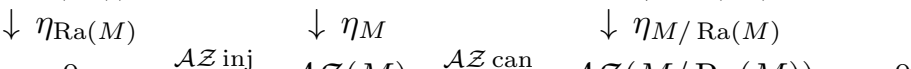

$$
\begin{aligned}
& 0 \rightarrow \quad 0 \quad \stackrel{\mathcal{A Z \text { inj }}}{\longrightarrow} \mathcal{A Z}(M) \stackrel{\mathcal{A Z \text { can }}}{\longrightarrow} \mathcal{A Z}(M / \operatorname{Ra}(M)) \rightarrow 0 \\
& \Longrightarrow \eta_{M}(\operatorname{Ra}(M))=0 \Longrightarrow \operatorname{Ra}(M) \subseteq \operatorname{ker}\left(\eta_{M}\right) \text {. }
\end{aligned}
$$


The closed modules can also be described by Morita equivalence, cf. [15, §IV.10, §XI.8]. The ( $\mathbf{Z}, \mathbf{A})$-bimodule $\epsilon_{\overline{0}} \mathbf{A}$ has the $\mathbf{Z}$-basis $\mathbf{v}:=\left(\epsilon_{\overline{0}}, \cdots, \epsilon_{\overline{0}} q^{N-1}\right)^{\top}$. If $R$ is any ring $R^{\text {op }}$ denotes the opposite ring of $R$, i.e., $R=R^{\text {op }}$ as abelian group and $r_{1}$ op $r_{2}=r_{2} r_{1}$. Then

$$
\operatorname{Hom}_{\mathbf{Z}}\left(\epsilon_{\overline{0}} \mathbf{A}, \epsilon_{\overline{0}} \mathbf{A}\right)^{\mathrm{op}} \cong \mathbf{B}:=\mathbf{Z}^{N \times N}, \alpha \mapsto b, \alpha(\mathbf{v})=b \mathbf{v},
$$

is an algebra isomorphism and induces the category equivalence

$$
\operatorname{Mod}_{\operatorname{Hom}_{\mathbf{Z}}\left(\epsilon_{\overline{0}} \mathbf{A}, \epsilon_{\overline{0}} \mathbf{A}\right)} \cong{ }_{\mathbf{B}} \operatorname{Mod}, M \mapsto M, b x=x \alpha, \alpha(\mathbf{v})=b \mathbf{v}, x \in M,
$$

between $\operatorname{Hom}_{\mathbf{Z}}\left(\epsilon_{\overline{0}} \mathbf{A}, \epsilon_{\overline{0}} \mathbf{A}\right)$-right and $\mathbf{B}$-left modules. Since $\epsilon_{\overline{0}} \mathbf{A}$ is a free generator of $\mathbf{z}$ Mod the Morita theorem [12, §18] yields the category equivalence

$$
\begin{aligned}
& \mathbf{z} \operatorname{Mod} \stackrel{\mathcal{A}}{\cong} \operatorname{Mod}_{\operatorname{Hom}_{\mathbf{Z}}\left(\epsilon_{\overline{0}} \mathbf{A}, \epsilon_{\overline{0}} \mathbf{A}\right)} \underset{(37)}{\cong} \quad{ }_{\mathbf{B}} \operatorname{Mod} \\
& P \quad \mapsto \quad \operatorname{Hom}_{\mathbf{Z}}\left(\epsilon_{\overline{0}} \mathbf{A}, P\right) \underset{(14)}{\stackrel{\mapsto}{\mapsto}} P^{N}=P^{N \times 1} .
\end{aligned}
$$

The structure of $P^{N}$ as $\mathbf{B}$-left module is given by the matrix multiplication

$$
(b, y) \mapsto b y, b \in \mathbf{B}=\mathbf{Z}^{N \times N}, y=\left(y_{0}, \cdots, y_{N-1}\right)^{\top} \in P^{N} .
$$

The structure of $\epsilon_{\overline{0}} \mathbf{A}$ as $\mathbf{A}$-right module induces the algebra homomorphisms

$$
\begin{aligned}
& \mathbf{A} \rightarrow \operatorname{Hom}_{\mathbf{Z}}\left(\epsilon_{\overline{0}} \mathbf{A}, \epsilon_{\overline{0}} \mathbf{A}\right)^{o p} \cong \mathbf{Z}^{N \times N} \\
& a \mapsto \quad\left(\epsilon_{\overline{0}} b \mapsto \epsilon_{\overline{0}} b a\right) \quad \mapsto \quad \rho(a) \quad \text { where } \\
& \mathbf{v} a=\rho(a) \mathbf{v}, \rho\left(\epsilon_{\bar{i}}\right)=\operatorname{diag}(0, \cdots, 0, \stackrel{i}{1}, 0, \cdots, 0), \rho(q)=\left(\begin{array}{ccccc}
0 & 1 & 0 & \cdots & 0 \\
0 & 0 & 1 & \cdots & 0 \\
\cdots & \cdots & \cdots & \cdots & \cdots \\
0 & 0 & 0 & \cdots & 1 \\
q^{N} & 0 & 0 & \cdots & 0
\end{array}\right) .
\end{aligned}
$$

Corollary 3.11. ( [9, Prop. 1])

$$
\rho\left(\epsilon_{\bar{k}} q^{l}\right)_{i j}= \begin{cases}1 & \text { if } i=k, j=k+l, k+l \leq N-1 \\ q^{N} & \text { if } i=k, j=k+l-n, k+l \geq N \\ 0 & \text { otherwise }\end{cases}
$$

With the standard basis $E_{k, l}, 0 \leq k, l \leq N-1$, of $\mathbb{Z}^{N \times N}$ this signifies

$$
\rho\left(\epsilon_{\bar{k}} q^{l}\right)=\left\{\begin{array}{ll}
E_{k, k+l} & \text { if } k+l \leq N-1 \\
q^{N} E_{k, k+l-N} & \text { if } k+l \geq N
\end{array} .\right.
$$

Example 3.12. Let $N=2$. Then

$$
\begin{gathered}
\rho\left(\epsilon_{0 z} q^{0}\right)=E_{0,0}=\left(\begin{array}{ll}
1 & 0 \\
0 & 0
\end{array}\right), \rho\left(\epsilon_{0} q^{1}\right)=E_{0,1}=\left(\begin{array}{ll}
0 & 1 \\
0 & 0
\end{array}\right), \\
\rho\left(\epsilon_{\overline{1}} q^{0}\right)=E_{1,0}=\left(\begin{array}{ll}
0 & 0 \\
1 & 0
\end{array}\right), \rho\left(\epsilon_{\overline{1}} q^{1}\right)=q^{2} E_{0,0}=q^{2}\left(\begin{array}{ll}
1 & 0 \\
0 & 0
\end{array}\right)
\end{gathered}
$$

Corollary 3.13. Consider the maps

$$
\begin{aligned}
& \mathbf{A} \stackrel{\eta_{\mathbf{A}}}{\longrightarrow} \quad \mathcal{A Z} \mathbf{A}=\left(\epsilon_{\overline{0}} \mathbf{A}\right)^{N} \quad \cong \mathbf{B}=\mathbf{Z}^{N \times N} \\
& a \mapsto \eta_{\mathbf{A}}(a)=\left(\begin{array}{c}
\epsilon_{\overline{0}} q^{0} a \\
\dddot{m}_{\overline{0}} q^{N-1} a
\end{array}\right)=\mathbf{v} a=\rho(a) \mathbf{v} \quad \mapsto \quad \rho(a)
\end{aligned}
$$

Since $\operatorname{ker}\left(\eta_{\mathbf{A}}\right)=\operatorname{Ra}(\mathbf{A})=0$ the maps $\eta_{\mathbf{A}}$ and thus also $\rho$ are injective, and hence $\mathbf{A}$ is a subalgebra of $\mathbf{B}$ via the explicitly given $\rho$ from Cor. 3.11. This corollary also implies that $\eta_{\mathbf{A}}$ and $\rho$ are not surjective and that hence $\mathbf{A}$ is not a closed $\mathbf{A}$-left module. 
Via $\rho$ every $\mathbf{B}$-module is also an $\mathbf{A}$-left module. If $P$ is any $\mathbf{Z}$-module then the A-structure of $P^{N}$ induced from $\rho$ is that of (15). Since $\mathcal{A}: P \mapsto P^{N}$ is a category equivalence from $\mathbf{Z}$ Mod both onto ${ }_{\left(\mathbf{A}, \epsilon_{\overline{0}}\right)}$ Mod and onto ${ }_{\mathbf{B}}$ Mod we conclude

Theorem 3.14. (i) The exact functors $\mathcal{Z}$ and $\mathcal{A}$ induce the category equivalence

$$
\mathcal{Z}:{ }_{\left(\mathbf{A}, \epsilon_{\overline{0}}\right)} \operatorname{Mod}={ }_{\mathbf{B}} \operatorname{Mod} \cong{ }_{\mathbf{Z}} \operatorname{Mod}: \mathcal{A}, M \mapsto \epsilon_{\overline{0}} M, P^{N} \leftarrow P .
$$

In particular, every closed $\mathbf{A}$-module $M$ is a $\mathbf{B}$-module where the $\mathbf{A}$ - and the $\mathbf{B}$ structures of $M$ are related by $\rho$.

(ii)

$$
\begin{aligned}
& \left({ }_{\mathbf{B}} \mathbf{M o d}^{\mathrm{fg}}\right)^{\mathrm{op}} \cong\left(\mathbf{z M o d}^{\mathrm{fg}}\right)^{\mathrm{op}} \cong\left\{\mathbf{A}^{\mathbb{N}} \text {-behaviors }\right\} \cong\left\{\mathbf{z}^{\mathbb{N} N} \text {-behaviors }\right\} \\
& M \quad \leftrightarrow \quad P \quad \leftrightarrow \quad \mathcal{B} \quad \leftrightarrow \quad \widehat{\mathcal{B}} \\
& P=\epsilon_{\overline{0}} M, M=P^{N}, \\
& \mathcal{B}=\operatorname{Hom}_{\mathbf{A}}\left(M, \mathbb{F}^{\mathbb{N}}\right)=\operatorname{Hom}_{\mathbf{B}}\left(M, \mathbb{F}^{\mathbb{N}}\right) \cong \widehat{\mathcal{B}}=\operatorname{Hom}_{\mathbf{Z}}\left(P, \mathbb{F}^{\mathbb{N} N}\right) .
\end{aligned}
$$

So the algebraic counter-part of the category of periodic behaviors, i.e., of $\mathbb{A}^{\mathbb{N}^{\mathbb{N}}}$ behaviors, is the category off.g. left B-modules and not that off.g. A-modules.

According to Thm. 3.14 the study of $\mathrm{A}^{\mathbb{N}} \mathbb{N}^{\mathbb{N}}$-behaviors requires that of f.g. Bmodules whereas f.g. A-modules are not needed. Properties of the latter are more complicated and were discussed in [9].

\section{System theory via Morita equivalence}

In this section we apply Thm. 3.14 and indeed discuss a slightly more general situation. Let $\mathbf{Z}$ be a commutative principal ideal domain and $\mathbf{Z}^{1 \times N}$ its standard progenerator with the standard basis $\mathbf{v} \in\left(\mathbf{Z}^{1 \times N}\right)^{N}$. We use the antiisomorphism (36)

$$
\mathbf{B}:=\mathbf{Z}^{N \times N} \cong \operatorname{Hom}_{\mathbf{Z}}\left(\mathbf{Z}^{1 \times N}, \mathbf{Z}^{1 \times N}\right)^{\mathrm{op}}, b \leftrightarrow \alpha, \alpha(\mathbf{v})=b \mathbf{v}
$$

and the Morita equivalence

$$
\mathcal{A}:{ }_{\mathbf{z}} \operatorname{Mod} \cong{ }_{\mathbf{B}} \operatorname{Mod}: \mathcal{Z}, P \rightarrow P^{N}, \epsilon_{\overline{0}} M \leftarrow M, \epsilon_{\overline{0}}:=\operatorname{diag}(1,0, \cdots, 0) .
$$

In particular, a B-left module is $\mathbf{B}$-f.g. if and only if it is $\mathbf{Z}$-f.g..

We also assume an injective cogenerator signal module ${ }_{\mathbf{z}} \widehat{W}$ and ${ }_{\mathbf{B}} W:=\widehat{W}^{N}$ that by equivalence is an injective cogenerator signal left $\mathbf{B}$-module.

Remark 4.1. The main, but not the only (see below) example for the preceding data is that from Thm. 3.14, i.e., $\mathbf{Z}=\mathbb{F}[\Delta]$ and $\mathbf{B}=\mathbf{Z}^{N \times N}$. We identify

$$
\begin{gathered}
\mathbf{Z}^{1 \times N}=\epsilon_{\overline{0}} \mathbf{A}=\oplus_{j=0}^{N-1} \mathbf{Z}_{\overline{0}} q^{j}, \mathbf{v}=\left(\epsilon_{\overline{0}} q^{0}, \cdots, \epsilon_{\overline{0}} q^{N-1}\right)^{\top}, \\
\mathbf{A}_{\text {Cor. 3.13 }}^{=} \rho(\mathbf{A}) \subset \mathbf{B}, a=\rho(a), \\
\epsilon_{\bar{i}}=\rho\left(\epsilon_{\bar{i}}\right)=\operatorname{diag}(0, \cdots, 0, \stackrel{i}{1}, 0, \cdots, 0), q=\rho(q)=\left(\begin{array}{ccccc}
0 & 1 & 0 & \cdots & 0 \\
0 & 0 & 1 & \cdots & 0 \\
\cdots & \cdots & \cdots & \cdots & \cdots \\
0 & 0 & 0 & \cdots & 1 \\
q^{N} & 0 & 0 & \cdots & 0
\end{array}\right) .
\end{gathered}
$$


In this case the basis vectors and the rows and columns of matrices in $\mathbf{Z}^{N \times N}$ are numbered from 0 to $N-1$ and we keep this numbering also in the more general situation of this section. The signal modules are

$$
\begin{gathered}
\widehat{W}=\mathbb{F}^{\mathbb{N} N} \text { and } W=\mathbb{F}^{\mathbb{N}} \underset{\text { ident. }}{=}\left(\mathbb{F}^{\mathbb{N} N}\right)^{N} \ni w \\
w=\left(w_{0}, \cdots, w_{N-1}\right)^{\top}, w(i+\tau)=w_{i}(\tau), i=0, \cdots, N-1, \tau \in \mathbb{N} N .
\end{gathered}
$$

The Morita functor $\mathcal{A}: P \mapsto P^{N}$ maps

$$
\mathbf{Z}^{1 \times N} \text { to }\left(\mathbf{Z}^{1 \times N}\right)^{N} \underset{\text { ident. }}{=} \mathbf{Z}^{N \times N}=\mathbf{B},
$$

where the left $\mathbf{B}$-structure of the left side is that from (39) and of $\mathbf{B}$ the canonical one. The Morita equivalence $\mathcal{A}$ preserves projectivity. In particular, for a f.g. $\mathbf{Z}$-module $P$ and $M=P^{N}$ one gets the equivalences

$P$ is $\mathbf{Z}$-torsionfree, i.e. $\mathbf{Z}$-free $\Longleftrightarrow M$ is $\mathbf{Z}$-free $\Longleftrightarrow M$ is $\mathbf{B}$-projective .

If (52) is satisfied the corresponding behavior $\operatorname{Hom}_{\mathbf{B}}(M, W) \cong \operatorname{Hom}_{\mathbf{Z}}(P, \widehat{W})$ is called controllable. Further the module $\mathbf{Z}^{N}=\mathcal{A} \mathbf{Z}$ is the unique indecomposable projective left $\mathbf{B}$-module, but not free. Every f.g. submodule of $\mathbf{Z}^{1 \times N k}$ is free of dimension $\leq N k$ and therefore every submodule of $\mathbf{B}^{1 \times k}$ is projective and the direct sum of at most $N k$ summands $\mathbf{Z}^{N}$, but not free in general.

Lemma 4.2. (i) A f.g. projective B-module $M$ is free if and only if $N^{2}$ divides $\operatorname{dim}_{\mathbf{Z}}(M)$.

(ii) If $\mathbf{B}^{1 \times k}=U_{1} \oplus U_{2}$ then $U_{1}$ is free if and only if $U_{2}$ is free and then $k=$ $\operatorname{dim}_{\mathbf{B}}\left(U_{1}\right)+\operatorname{dim}_{\mathbf{B}}\left(U_{2}\right)$.

Proof. Since $\epsilon_{\overline{0}} \mathbf{B}=\mathbf{Z}^{1 \times N}$ and $\operatorname{dim}_{\mathbf{Z}}\left(\mathbf{Z}^{1 \times N}\right)=N$ a f.g. projective $\mathbf{B}$-module $M$ is free if and only if $N$ divides $\operatorname{dim}_{\mathbf{Z}}\left(\epsilon_{\overline{0}} M\right)$ or $N^{2}$ divides $\operatorname{dim}_{\mathbf{Z}}(M)=N \operatorname{dim}_{\mathbf{Z}}\left(\epsilon_{\overline{0}} M\right)$. (ii) Due to $\operatorname{dim}_{\mathbf{Z}}(\mathbf{B})=N^{2}$ (ii) follows directly from (i).

The functor $\mathcal{A}$ maps a free $\mathbf{Z}$-module of dimension divisible by $N$ onto a free $\mathbf{B}$ module, especially

$$
\mathcal{A}: \mathbf{Z}^{1 \times N k}=\left(\mathbf{Z}^{1 \times N}\right)^{1 \times k}=\mathbf{Z}^{1 \times(N \times k)} \mapsto\left(\left(\mathbf{Z}^{1 \times N}\right)^{1 \times k}\right)^{N}=\left(\mathbf{Z}^{N \times N}\right)^{1 \times k} .
$$

Notice that the identification $\mathbf{Z}^{1 \times N k}=\left(\mathbf{Z}^{1 \times N}\right)^{1 \times k}$ requires to divide the numbers $1, \cdots, N k$ into $k$ blocks of length $N$. Such a division is either adapted to the context or chosen arbitrarily. For two such modules there is the isomorphism

$$
\begin{aligned}
& \operatorname{Hom}_{\mathbf{Z}}\left(\left(\mathbf{Z}^{1 \times N}\right)^{1 \times r},\left(\mathbf{Z}^{1 \times N}\right)^{1 \times k}\right) \cong \mathbf{B}^{r \times k}, \varphi=\circ R \leftrightarrow R, \varphi(\eta)=\eta R=: \xi, \\
& \text { where } R=(R(i, j))_{i, j} \in \mathbf{B}^{r \times k}, R(i, j) \in \mathbf{B}, \\
& \eta=(\eta(1), \cdots, \eta(r)), \eta(i) \in \mathbf{Z}^{1 \times N}, \xi=(\xi(1), \cdots, \xi(k)), \xi(j) \in \mathbf{Z}^{1 \times N} \\
& \xi=\varphi(\eta)=\eta R, \xi(j)=\sum_{i=1}^{r} \eta(i) R(i, j) .
\end{aligned}
$$


This isomorphism preserves products, i.e., transforms the product of composable maps into the matrix product. The isomorphism

$$
\begin{gathered}
\operatorname{Hom}_{\mathbf{B}}\left(\mathbf{B}^{1 \times r}, \mathbf{B}^{1 \times k}\right) \cong \mathbf{B}^{r \times k}, \Phi=\circ R \leftrightarrow R, \Phi(Y)=Y R=: X, \\
Y=(Y(1), \cdots, Y(r)), X=(X(1), \cdots, X(k)), X=Y R, X(j)=\sum_{i=1}^{r} Y(i) R(i, j),
\end{gathered}
$$

is the natural one. The equivalence functor $\mathcal{A}$ maps the $\mathbf{Z}$-linear map $\circ R$ onto the B-linear map $\circ R$, more precisely

$$
\begin{aligned}
\mathcal{A}\left(\circ R:\left(\mathbf{Z}^{1 \times N}\right)^{1 \times r} \rightarrow\left(\mathbf{Z}^{1 \times N}\right)^{1 \times k}\right)=\left(\circ R:\left(\mathbf{Z}^{1 \times N}\right)^{1 \times r} \rightarrow\left(\mathbf{Z}^{1 \times N}\right)^{1 \times k}\right)^{N} \\
\quad=\circ R: \mathbf{B}^{1 \times r} \rightarrow \mathbf{B}^{1 \times k} .
\end{aligned}
$$

In the sequel we therefore identify

$$
\begin{aligned}
& \mathbf{B}^{r \times k}=\operatorname{Hom}_{\mathbf{B}}\left(\mathbf{B}^{1 \times r}, \mathbf{B}^{1 \times k}\right)=\operatorname{Hom}_{\mathbf{Z}}\left(\left(\mathbf{Z}^{1 \times N}\right)^{1 \times r},\left(\mathbf{Z}^{1 \times N}\right)^{1 \times k}\right), \\
& \text { in particular } \mathbf{B}^{k \times k}=\mathbf{Z}^{N k \times N k} \supset \mathrm{Gl}_{k}(\mathbf{B})=\mathrm{Gl}_{N k}(\mathbf{Z}) \\
& =\left\{R \in \mathbf{Z}^{N k \times N k} ; \operatorname{det}(R) \in \mathrm{U}(\mathbf{Z})\right\} \\
& \mathcal{A}\left(\mathbf{Z}^{1 \times N r} R\right)=\left(\mathbf{Z}^{1 \times N r} R\right)^{N}=\mathbf{B}^{1 \times r} R \subseteq \mathbf{B}^{1 \times k} \\
& \operatorname{dim}_{\mathbf{Z}}\left(\mathbf{B}^{1 \times r} R\right)=N \operatorname{dim}_{\mathbf{Z}}\left(\mathbf{Z}^{1 \times N r} R\right)=N \operatorname{rank}_{\mathbf{Z}}(R) .
\end{aligned}
$$

Here $\mathrm{U}(\mathbf{Z})$ is the group of units or invertible elements of $\mathbf{Z}$.

Remark 4.3. The preceding identification (57) implies in particular that the Smith form of matrices in $\mathbf{Z}^{N r \times N k}$ can be applied to $R \in \mathbf{B}^{r \times k}$ and that $R$ is equivalent to a block matrix $\left(\begin{array}{cc}D & 0 \\ 0 & 0\end{array}\right)$ where $D$ is a diagonal matrix of $\mathbf{Z}$-rank $l=\operatorname{rank}_{\mathbf{Z}}(R)$. If $l=$ $m N+n, m, n \in \mathbb{N}, n<N$, one can assume moreover that $D=\operatorname{diag}\left(d_{1}, \cdots, d_{m}, d_{m+1}\right)$ where the $d_{\mu}$ are diagonal in $\mathbf{B}=\mathbf{Z}^{N \times N}$ and of full $\mathbf{Z}$-rank $N$ or regular (nonzerodivisors) in $\mathbf{B}$ for $\mu \leq m$. The row module $\mathbf{B}^{1 \times r} R \subset \mathbf{B}^{1 \times k}$ is always projective, but $\mathbf{B}$-free only if the $\mathbf{Z}$-rank $l=m N+n$ is divisible by $N$ or $n=0$ and hence $d_{m+1}=0$. Then $R$ is row-equivalent to a matrix $R^{\prime} \in \mathbf{B}^{m \times k}$ whose rows are a $\mathbf{B}$-basis of the row-module $\mathbf{B}^{1 \times r} R=\mathbf{B}^{1 \times m} R^{\prime}$.

For $b \in \mathbf{B}=\mathbf{Z}^{N \times N}$ and $w=\left(w_{0}, \cdots, w_{N-1}\right)^{\top} \in W=\widehat{W}^{N}$ the action of $b$ on $w$ from (39) is defined by

$$
b \circ w=\left(\sum_{\nu=0}^{N-1} b_{\mu \nu} \circ w_{\nu}\right)_{0 \leq \mu \leq N-1} .
$$

More generally we get

$$
\begin{gathered}
W^{k}=\left(\widehat{W}^{N}\right)^{k}=\widehat{W}^{N k} \ni w=\left(w_{1}, \cdots, w_{k}\right)^{\top}, \\
w_{j}=\left(w_{j, 0}, \cdots, w_{j, N-1}\right) \in W=\widehat{W}^{N} .
\end{gathered}
$$

The matrix $R \in \mathbf{B}^{r \times k}$ from (54) induces the $\mathbf{Z}$-linear system map

$$
\begin{gathered}
\circ R: W^{k}=\widehat{W}^{N k} \rightarrow W^{r}=\widehat{W}^{N r}, w \mapsto R \circ w,(R \circ w)_{i}=\sum_{j=1}^{k} R(i, j) \circ w_{j}, \\
(R \circ w)_{i, \mu}=\sum_{j=1}^{k} \sum_{\nu=0}^{N-1} R(i, j)_{\mu \nu} \circ w_{j, \nu}
\end{gathered}
$$


The associated behavior is

$$
\begin{gathered}
\mathcal{B}=\left\{w \in W^{k}=\widehat{W}^{N k} ; R \circ w=0\right\} \underset{\text { Malgrange }}{\cong} \\
\operatorname{Hom}_{\mathbf{B}}\left(\mathbf{B}^{1 \times k} / \mathbf{B}^{1 \times r} R, W\right) \underset{\text { Morita }}{\cong} \operatorname{Hom}_{\mathbf{Z}}\left(\mathbf{Z}^{1 \times N k} / \mathbf{Z}^{1 \times N r} R, \widehat{W}\right) .
\end{gathered}
$$

The behavior $\mathcal{B}$ is thus a ${ }_{\mathbf{B}} W$ - and a $\mathbf{z} \widehat{W}$-behavior. Interpreted as the latter it is an LTI behavior and the standard LTI systems theory for the injective cogenerator signal module $\mathbf{z} \widehat{W}$, for instance for $\mathbb{F}[\Delta] \mathbb{F}^{\mathbb{N} N}$, can be applied to it. The system factor modules of the behavior $\mathcal{B}$ appear in the exact sequences

$$
\begin{aligned}
& \mathbf{B}^{1 \times r} \quad \stackrel{\circ R}{\longrightarrow} \quad \mathbf{B}^{1 \times k} \quad \stackrel{\text { can }}{\longrightarrow} \quad M \quad \rightarrow 0 \\
& \left(\mathbf{Z}^{1 \times N}\right)^{1 \times r} \stackrel{\circ R}{\longrightarrow}\left(\mathbf{Z}^{1 \times N}\right)^{1 \times k} \stackrel{\text { can }}{\longrightarrow} \epsilon_{\overline{0}} M \quad \rightarrow 0 \\
& \text { where } M:=\mathbf{B}^{1 \times k} / \mathbf{B}^{1 \times r} R=\left(\epsilon_{\overline{0}} M\right)^{N}, \epsilon_{\overline{0}} M=\mathbf{Z}^{1 \times N k} / \mathbf{Z}^{1 \times N r} R
\end{aligned}
$$

and where can denotes the canonical map onto the factor module.

It is obvious that the preceding considerations can be applied to all matrices $R \in \mathbf{B}^{r \times k}$ and therefore to all ${ }_{\mathbf{B}} W$-behaviors and especially to all periodic ${ }_{\mathbf{A}} \mathbb{F}^{\mathbb{N}}$-behaviors, but not to all $\mathbf{z} \widehat{W}$-behaviors because the number of rows and columns of $R$ as a matrix with entries in $\mathbf{Z}$ were assumed to be multiples of $N$.

The quotient field

$$
\mathbf{K}:=\mathbf{Z}_{\mathbb{S}_{\mathbf{Z}}}=\left\{s^{-1} a ; s \in \mathbb{S}_{\mathbf{Z}}, a \in \mathbf{Z}\right\}, \mathbb{S}_{\mathbf{Z}}:=\mathbf{Z} \backslash\{0\} \subset \mathbf{Z}
$$

plays an important part in the LTI theory and thus here too. For $\mathbf{Z}=\mathbb{F}[\Delta]$ it is the field $\mathbb{F}(\Delta)$ of rational functions. It gives rise to the quotient ring

$$
\mathbf{Q}:=\mathbf{B}_{\mathbb{S}_{\mathbf{Z}}}=\mathbf{K} \otimes_{\mathbf{Z}} \mathbf{B}=\left\{s^{-1} b ; s \in \mathbb{S}_{\mathbf{Z}}, b \in \mathbf{B}\right\}=\mathbf{K}^{N \times N}
$$

that is a simple artinian $\mathbf{K}$-algebra. By the standard LTI theory the $\mathbf{z} \widehat{W}$-behavior $\mathcal{B}$ from (61) is autonomous if and only if $\epsilon_{\overline{0}} M$ is a torsion module or, equivalently, $\epsilon_{\overline{0}} M_{\mathbb{S}_{\mathbf{Z}}}=0$. The ${ }_{\mathbf{B}} W$-behavior $\mathcal{B}$ is called autonomous if and only it is such as LTI behavior, cf. [5, §4.3]. According to (62) this means that $M$ is a $\mathbf{Z}$-torsion module or $M_{\mathbb{S}_{\mathbf{Z}}}=0$. For $\mathbf{Z}=\mathbb{F}[\Delta]$ autonomy is also equivalent to the $\mathbb{F}$-finite dimensionality of $M$ and $\epsilon_{\overline{0}} M$. For the signal module ${ }_{\mathbf{B}} \mathbb{F}^{\mathbb{N}}$ it also means that the trajectories in $\mathcal{B}$ are determined by initial conditions in the following sense: There is a number $d \in \mathbb{N}$ such that the initial projection

$$
\mathcal{B} \rightarrow\left(\mathbb{F}^{\mathbb{N}}\right)^{d k}, w \mapsto(w(0), \cdots, w(d-1))^{\top},
$$

is injective.

Input/output $(I O)$ structures of the ${ }_{\mathbf{B}} W$-behavior $\mathcal{B}$ are defined in the following fashion: Let $\delta_{j}, j=1, \cdots, k$, be the standard basis of $\mathbf{B}^{1 \times k}$ and

$$
\mathbf{w}:=\left(\delta_{1}+\mathbf{B}^{1 \times r} R, \cdots, \delta_{k}+\mathbf{B}^{1 \times r} R\right)^{\top} \in M^{k}
$$

the canonical set of $\mathbf{B}$-generators of $M$. An IO-structure of $M$ is given by a subfamily $\mathbf{u}=\left(\mathbf{u}_{1}, \cdots, \mathbf{u}_{m}\right)^{\top} \in M^{m}$ of $\mathbf{w}$ such that the $\mathbf{u}_{i}$ are $\mathbf{B}$-linearly independent and $M / \mathbf{B}^{1 \times m} \mathbf{u}$ is a $\mathbf{Z}$-torsion module. After the usual permutation of the $\mathbf{w}_{j}$ we assume that

$$
\mathbf{w}=\left(\begin{array}{l}
\mathbf{y} \\
\mathbf{u}
\end{array}\right) \in M^{p+m}, \mathbf{y}=\left(\mathbf{y}_{1}, \cdots, \mathbf{y}_{p}\right)^{\top}, p+m=k .
$$


Correspondingly the matrix $R$ and $\mathcal{B}$ are decomposed as

$$
R=\left(D_{l},-N_{l}\right) \in \mathbf{B}^{r \times(p+m)} \text { and } \mathcal{B}=\left\{\left(\begin{array}{c}
y \\
u
\end{array}\right) \in W^{p+m} ; D_{l} \circ y=N_{l} \circ u\right\} .
$$

The IO-property of $\left(\begin{array}{l}\mathbf{y} \\ \mathbf{u}\end{array}\right)$ can be alternatively characterized as follows:

Lemma 4.4. For $\left(\begin{array}{l}\mathbf{y} \\ \mathbf{u}\end{array}\right) \in M^{p+m}$ as above and $M^{0}:=\mathbf{B}^{1 \times p} / \mathbf{B}^{1 \times r} D_{l}$ the following properties are equivalent:

(i) The decomposition $\mathbf{w}=\left(\begin{array}{l}\mathbf{y} \\ \mathbf{u}\end{array}\right)$ is an IO-decomposition.

(ii) The sequence

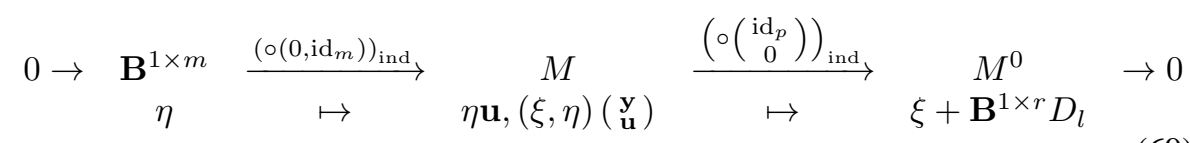

is exact and $M^{0}$ is a $\mathbf{Z}$-torsion module.

(iii) The projection $\mathcal{B} \rightarrow W^{m}, w=\left(\begin{array}{l}y \\ u\end{array}\right) \mapsto u$, is surjective and the ${ }_{\mathrm{B}} W$-behavior $\mathcal{B}^{0}:=\left\{y \in W^{p} ; D_{l} \circ y=0\right\}$ is autonomous.

(iv) The induced map $\mathbf{Q}^{1 \times m}=\mathbf{B}_{S_{\mathbf{Z}}}^{1 \times m} \rightarrow M_{\mathbb{S}_{\mathbf{Z}}}, \delta_{i} \mapsto \frac{\mathbf{u}_{i}}{1}$, is a $\mathbf{Q}$-isomorphism.

(v) The submodule $\mathbf{B}^{1 \times r}\left(D_{l},-N_{l}\right)$ is free of dimension $p$ and $D_{l}$ has a left inverse in $\mathbf{Q}^{p \times r}$.

(vi) $\operatorname{rank}_{\mathbf{Z}}\left(D_{l}\right)=\operatorname{rank}_{\mathbf{Z}}(R)=p N$, i.e., $\left(D_{l},-N_{l}\right) \in \mathbf{B}^{r \times(p+m)}=\mathbf{Z}^{N r \times N(p+m)}$ defines an IO-decomposition of the $\mathbf{z} \widehat{W}$-behavior $\mathcal{B}$.

Since $\mathbf{B}^{1 \times r} R=\mathbf{B}^{1 \times r}\left(D_{l},-N_{l}\right)$ is free of dimension $p$ we may always assume $r=p$ w.l.o.g.. In this case $D_{l} \in \mathrm{Gl}_{p}(\mathbf{Q})=\mathrm{Gl}_{N p}(\mathbf{K})$ and the matrix

$$
G=D_{l}^{-1} N_{l} \in \mathbf{Q}^{p \times m}=\mathbf{K}^{N p \times N m}
$$

is the transfer matrix of the IO-behavior $\mathcal{B}$. It is characterized by the equation $\mathbf{Q}^{1 \times r} R=$ $\mathbf{Q}^{1 \times p}\left(\mathrm{id}_{p},-G\right)$.

Proof. $(i) \Longleftrightarrow(i i)$ : The exactness of (69) without the 0 on the left is standard and the remaining properties in (ii) are precisely the conditions of (i).

$($ ii $) \Longleftrightarrow($ iii $)$ : by duality since ${ }_{\mathbf{B}} W$ is an injective cogenerator.

$($ iii $) \Longleftrightarrow(i v)$ : This follows from the exactness of $M \mapsto M_{\mathbb{S}_{\mathbf{z}}}$ and the fact that $M^{0}$ is $\mathbf{Z}$-torsion if and only if $M_{\mathbb{S}_{\mathbf{Z}}}^{0}=0$.

$(i v) \Longrightarrow(v)$ : (a) The isomorphism from (iv) implies

$$
\begin{aligned}
& \mathbf{Q}^{1 \times m} \cong M_{\mathbb{S}_{\mathbf{Z}}} \cong \mathbf{Q} \otimes_{\mathbf{B}} M \cong \mathbf{Q}^{1 \times k} / \mathbf{Q}^{1 \times r} R \\
& \Longrightarrow \operatorname{dim}_{\mathbf{Z}}\left(\mathbf{B}^{1 \times r} R\right)=\operatorname{dim}_{\mathbf{K}}\left(\mathbf{Q}^{1 \times r} R\right)=\operatorname{dim}_{\mathbf{K}}\left(\mathbf{Q}^{1 \times k}\right)-\operatorname{dim}_{\mathbf{K}}\left(\mathbf{Q}^{1 \times m}\right) \\
&=(k-m) N^{2}=p N^{2} \Longrightarrow \mathbf{B}^{1 \times r} R \text { free }, \operatorname{dim}_{\mathbf{B}}\left(\mathbf{B}^{1 \times r} R\right)=p .
\end{aligned}
$$

(b) The torsion property of $M^{0}$ implies

$$
0=M_{\mathbb{S}_{\mathbf{Z}}}^{0} \cong \mathbf{Q} \otimes_{\mathbf{B}} M^{0} \cong \mathbf{Q}^{1 \times p} / \mathbf{Q}^{1 \times r} D_{l} .
$$

This is equivalent to the existence of a left inverse of $D_{l}$ in $\mathbf{Q}^{p \times r}$.

$(v) \Longrightarrow(i v)$ : As in $(i v) \Longrightarrow(v)$ we conclude that $M^{0}$ is $\mathbf{Z}$-torsion. This implies that

$$
\mathbf{Q}^{1 \times m} \rightarrow M_{\mathbb{S}_{\mathbf{Z}}} \cong \mathbf{Q}^{1 \times k} / \mathbf{Q}^{1 \times r} R
$$

is surjective. Dimension count furnishes

$$
\begin{aligned}
& \operatorname{dim}_{\mathbf{K}}\left(\mathbf{Q}^{1 \times k} / \mathbf{Q}^{1 \times r} R\right)=\operatorname{dim}_{\mathbf{K}}\left(\mathbf{Q}^{1 \times k}\right)-\operatorname{dim}_{\mathbf{K}}\left(\mathbf{Q}^{1 \times r} R\right) \\
& =N^{2} k-N^{2} p=N^{2} m=\operatorname{dim}_{\mathbf{K}}\left(\mathbf{Q}^{1 \times m}\right) .
\end{aligned}
$$


The dimension equality over the field $\mathbf{K}$ implies that the surjection (71) is bijective. $(i v) \Longleftrightarrow(v i)$ : By standard LTI theory the IO-property of the $\mathbf{z} \widehat{W}$-behavior $\mathcal{B}$ is equivalent to the isomorphy

$$
\left(\circ\left(0, \operatorname{id}_{N m}\right)\right)_{\text {ind }}: \mathbf{K}^{1 \times N m} \cong \mathbf{K}^{1 \times N k} / \mathbf{K}^{1 \times N r}\left(D_{l},-N_{l}\right) .
$$

By Morita equivalence this isomorphism is equivalent to the isomorphism from (iv).

Corollary and Definition 4.5. Assume w.l.o.g. that $r=p$ in Lemma 4.4. Then the standard sequence

$$
0 \rightarrow \mathbf{B}^{1 \times p} \stackrel{\circ\left(D_{l},-N_{l}\right)}{\longrightarrow} \mathbf{B}^{1 \times(p+m)} \stackrel{\text { can }}{\longrightarrow} M \rightarrow 0
$$

is exact and the following properties are equivalent:

(i) $\left(D_{l},-N_{l}\right)$ has a right inverse.

(ii) $M$ is $\mathbf{Z}$-free and then indeed $\mathbf{B}$-free of dimension $m$.

The representation $G=D_{l}^{-1} N_{l}$ is then called a left coprime factorization of $G$.

Proof. By Morita equivalence $\mathbf{Z}$-freeness and B-projectivity of $M$ coincide. Equation (72) implies that (i) is equivalent to the existence of a direct decomposition $\mathbf{B}^{1 \times(p+m)} \cong$ $\mathbf{B}^{1 \times p} \times M$ or the projectivity of $M$. Lemma 4.2 implies that $M$ is $\mathbf{B}$-free with $\operatorname{dim}_{\mathbf{B}}(M)=m$.

Recall that the behavior $\mathcal{B} \cong \operatorname{Hom}_{\mathbf{Z}}\left(\epsilon_{\overline{0}} M, \widehat{W}\right) \cong \operatorname{Hom}_{\mathbf{B}}(M, W)$ is controllable as LTI, resp. as periodic behavior if and only if $M$ is $\mathbf{Z}$-free, resp. B-projective.

Remark 4.6. Lemma 4.4 shows that an IO-decomposition of the ${ }_{\mathbf{B}} W$-behavior is also one of the $\mathbf{z} \widehat{W}$-behavior $\mathcal{B}$, but there are many more IO-decomposition of $\mathcal{B}$ as $\mathbf{z} \widehat{W}$ behavior than as ${ }_{\mathbf{B}} W$-behavior. Whereas an arbitrary LTI behavior admits at least one IO-decomposition this is not true for periodic behaviors since already the necessary condition that the projective module $\mathbf{B}^{1 \times r} R$ be free need not be satisfied. But even if $\mathbf{B}^{1 \times r} R$ is free of dimension $p$ and $r=p$ w.l.o.g. there need not be a decomposition $R=\left(D_{l},-N_{l}\right) \in \mathbf{B}^{p \times(p+m)}$ (after a suitable column permutation) with $D_{l} \in \mathrm{Gl}_{p}(\mathbf{Q})$, for instance in

$$
N=2 \text { and } R=\left(\left(\begin{array}{ll}
1 & 2 \\
0 & 0
\end{array}\right),\left(\begin{array}{ll}
0 & 0 \\
1 & 3
\end{array}\right)\right)=\left(\begin{array}{llll}
1 & 2 & 0 & 0 \\
0 & 0 & 1 & 3
\end{array}\right) \in \mathbf{B}^{1 \times 2}=\mathbf{Z}^{2 \times 4} .
$$

So the IO-decomposition of a periodic behavior is an essential additional structure. In [1, Def. 53] the authors define an IO-decomposition of $\mathcal{B}$ as one of the LTI behavior, but for the time-axis $\mathbb{Z}$ instead of $\mathbb{N}$ here. For the further considerations in the present paper this notion is too weak.

Lemma 4.7. Assume that $R=\left(D_{l}^{0},-N_{l}^{0}\right) \in \mathbf{B}^{p \times(p+m)}$ with $D_{l}^{0} \in \mathrm{Gl}_{p}(\mathbf{Q})$ and free $M:=\mathbf{B}^{1 \times(p+m)} / \mathbf{B}^{1 \times p}\left(D_{l}^{0},-N_{l}^{0}\right)$. In other words, $R=\left(D_{l}^{0},-N_{l}^{0}\right)$ is an IOdecomposition and $G:=\left(D_{l}^{0}\right)^{-1} N_{l}^{0}$ is a left coprime factorization. Then there are matrices $D_{r}^{0}, N_{r}^{0}, R_{l}^{0}, S_{l}^{0}, R_{r}^{0}, S_{r}^{0} \in \mathbf{B}^{\bullet \times \bullet}$ of suitable sizes with the following properties: 
(i) The following two sequences are exact,

$$
\begin{aligned}
& 0 \rightarrow \mathbf{B}^{1 \times p} \stackrel{\circ\left(D_{l}^{0},-N_{l}^{0}\right)}{\longrightarrow} \mathbf{B}^{1 \times(p+m)} \stackrel{\circ\left(\begin{array}{c}
N_{r}^{0} \\
D_{r}^{0}
\end{array}\right)}{\longrightarrow} \mathbf{B}^{1 \times m} \rightarrow 0 \\
& 0 \leftarrow \mathbf{B}^{1 \times p} \stackrel{\circ\left(\begin{array}{c}
S_{r}^{0} \\
-R_{r}^{0}
\end{array}\right)}{\longleftarrow} \mathbf{B}^{1 \times(p+m)} \stackrel{\circ\left(R_{l}^{0}, S_{l}^{0}\right)}{\longleftarrow} \mathbf{B}^{1 \times m} \leftarrow 0,
\end{aligned}
$$

in particular $D_{l}^{0} G=N_{l}^{0}$ and $G D_{r}^{0}=N_{r}^{0}$.

(ii) $\mathbf{B}^{1 \times p}\left(D_{l}^{0},-N_{l}^{0}\right)=\operatorname{ker}\left(\circ\left(\begin{array}{c}G \\ \operatorname{id}_{m}\end{array}\right): \mathbf{B}^{1 \times(p+m)} \rightarrow \mathbf{Q}^{1 \times m}\right)$. This shows that the left coprime factorization $G=\left(D_{l}^{0}\right)^{-1} N_{l}^{0}$ is unique up to row equivalence of $\left(D_{l}^{0},-N_{l}^{0}\right)$

(iii) The following matrix equations hold:

$$
\begin{gathered}
\left(D_{l}^{0},-N_{l}^{0}\right)\left(\begin{array}{c}
S_{r}^{0} \\
-R_{r}^{0}
\end{array}\right)=\mathrm{id}_{p},\left(R_{l}^{0}, S_{l}^{0}\right)\left(\begin{array}{c}
N_{r}^{0} \\
D_{r}^{0}
\end{array}\right)=\mathrm{id}_{m}, D_{r}^{0} \in \mathrm{Gl}_{m}(\mathbf{Q}) \\
\left(\begin{array}{cc}
D_{l}^{0} & -N_{l}^{0} \\
R_{l}^{0} & S_{l}^{0}
\end{array}\right)\left(\begin{array}{cc}
S_{r}^{0} & N_{r}^{0} \\
-R_{r}^{0} & D_{r}^{0}
\end{array}\right)=\left(\begin{array}{cc}
\operatorname{id}_{p} & 0 \\
0 & \operatorname{id}_{m}
\end{array}\right)=\mathrm{id}_{p+m},\left(\begin{array}{cc}
D_{l}^{0} & -N_{l}^{0} \\
R_{l}^{0} & S_{l}^{0}
\end{array}\right)^{-1}=\left(\begin{array}{cc}
S_{r}^{0} & N_{r}^{0} \\
-R_{r}^{0} & D_{r}^{0}
\end{array}\right) . \\
\hline 74)
\end{gathered}
$$

Then $G=N_{r}^{0}\left(D_{r}^{0}\right)^{-1}$ is called a right coprime factorization of $G$ that is also unique up to column equivalence of $\left(\begin{array}{c}N_{r}^{0} \\ D_{r}^{0}\end{array}\right)$.

(iv) All other quadrupels $S_{l}, R_{l}, R_{r}, S_{r} \in \mathbf{B}^{\bullet} \times \bullet$ with the properties from (i) and (iii) (without the index 0 ) are obtained with arbitrary $X \in \mathbf{B}^{m \times p}$ by

$$
\left(\begin{array}{c}
S_{r} \\
-R_{r}
\end{array}\right)=\left(\begin{array}{c}
S_{r}^{0} \\
-R_{r}^{0}
\end{array}\right)-\left(\begin{array}{c}
N_{r}^{0} \\
D_{r}^{0}
\end{array}\right) X,\left(R_{l}, S_{l}\right)=\left(R_{l}^{0}, S_{l}^{0}\right)+X\left(D_{l}^{0},-N_{l}^{0}\right) .
$$

This is a variant of the famous Kučera-Youla parametrization.

(v) Generically (in the Zariski topology of $\mathbf{B}^{m \times p}=\mathbf{Z}^{N m \times N p}$ ) or for almost all $X$ the additional inclusions $S_{l} \in \mathrm{Gl}_{m}(\mathbf{Q})$ and $S_{r} \in \mathrm{Gl}_{p}(\mathbf{Q})$ hold. This means that $\left(R_{l}, S_{l}\right)$ is also an IO-decomposition.

Proof. Since $M$ is free of dimension $m$ equation (72) and replacement of $M$ by $\mathbf{B}^{1 \times m}$ furnish the first exact sequence in (73). The remaining assertions are elementary algebra [16, Ch. 4], [3, Lemmas 2.3, 3.10]. The proof of $D_{r}^{0} \in \mathrm{Gl}_{m}(\mathbf{Q})$ follows from $\mathrm{id}_{m}=R_{l} N_{r}^{0}+S_{l} D_{r}^{0}=\left(R_{l} G+S_{l}\right) D_{r}^{0}$. The first exact sequence in (73) and $\left(\begin{array}{c}N_{r}^{0} \\ D_{r}^{0}\end{array}\right)=\left(\begin{array}{c}G \\ \operatorname{id}_{m}\end{array}\right) D_{r}^{0}$ imply (ii).

Lemma 4.8. An arbitrary matrix $G \in \mathbf{Q}^{p \times m}$ admits a left coprime factorization $G=$ $\left(D_{l}^{0}\right)^{-1} N_{l}^{0}$ as in Cor. 4.5 that, in turn, gives rise to all data of Lemma 4.7, in particular to the right coprime factorization $G=N_{r}^{0}\left(D_{r}^{0}\right)^{-1}$.

Proof. Item (ii) of Lemma 4.7 suggests to define

$$
\begin{aligned}
& U:=\operatorname{ker}\left(\circ\left(\begin{array}{c}
G \\
\operatorname{id}_{m}
\end{array}\right): \mathbf{B}^{1 \times(p+m)} \rightarrow \mathbf{Q}^{1 \times m}\right) \\
& \Longrightarrow M:=\mathbf{B}^{1 \times(p+m)} / U \underset{\text { ident. }}{=} \mathbf{B}^{1 \times(p+m)}\left(\begin{array}{c}
G \\
\operatorname{id}_{m}
\end{array}\right) \subseteq \mathbf{Q}^{1 \times m} .
\end{aligned}
$$


All f.g. $\mathbf{B}$-submodules of the $\mathbf{K}$-space $\mathbf{Q}^{1 \times m}$ are $\mathbf{Z}$-torsionfree and $\mathbf{B}$-projective. Hence $M$ is B-projective and

$$
\begin{aligned}
& M_{\mathbb{S}_{\mathbf{Z}}}=\mathbf{Q}^{1 \times(p+m)}\left(\begin{array}{c}
G \\
\operatorname{id}_{m}
\end{array}\right)=\mathbf{Q}^{1 \times m} \\
& \Longrightarrow \operatorname{dim}_{\mathbf{Z}}(M)=\operatorname{dim}_{\mathbf{K}}\left(M_{\mathbb{S}_{\mathbf{Z}}}\right)=\operatorname{dim}_{\mathbf{K}}\left(\mathbf{Q}^{1 \times m}\right)=N^{2} m
\end{aligned}
$$

We infer that $M$ is $\mathbf{B}$-free of dimension $m$ and induces a direct decomposition $\mathbf{B}^{1 \times(p+m)} \cong$ $U \times M$. This and Lemma 4.2 , in turn, imply that $U$ is $\mathbf{B}$-free of dimension $p$ and thus of the form

$$
\begin{aligned}
& U=\mathbf{B}^{1 \times p}\left(D_{l}^{0},-N_{l}^{0}\right) \text { with } \operatorname{dim}_{\mathbf{B}}(U)=p, D_{l}^{0} G=N_{l}^{0} \text { and }\left(D_{l}^{0},-N_{l}^{0}\right)=D_{l}^{0}\left(\operatorname{id}_{p},-G\right) \\
& \Longrightarrow N p=\operatorname{rank}_{\mathbf{Z}}\left(D_{l}^{0},-N_{l}^{0}\right)=\operatorname{rank}_{\mathbf{Z}}\left(D_{l}^{0}\right) .
\end{aligned}
$$

With Lemma 4.4,(vi), we conclude that $\left(D_{l}^{0},-N_{l}^{0}\right)$ is an IO-decomposition and with Cor. 4.5,(ii), that $G=\left(D_{l}^{0}\right)^{-1} N_{l}^{0}$ is the left coprime factorization, unique up to row equivalence.

\section{Stabilizing controllers}

In this section we construct and parametrize all stabilizing controllers of an $I O-\mathbb{F}[\Delta]^{N \times N} \mathbb{F}^{\mathbb{N}}$ behavior by reduction to the LTI case.

The assumptions and notations of Section 4 remain in force with the specialization to the data from Section 3. In addition we assume the base field $\mathbb{F}:=\mathbb{R}, \mathbb{C}$ of real or complex numbers, the polynomial algebra $\mathbf{Z}=\mathbb{F}[\Delta]$ and the subalgebra $\mathbb{F}(\Delta)_{\text {pr }} \subset$ $\mathbf{K}=\mathbb{F}(\Delta)$ of proper rational functions [16, Ch. 2]. The relevant signal spaces are $\mathbb{F}[\Delta] \widehat{W}:=\mathbb{F}^{\mathbb{N} N}$ and ${ }_{\mathbf{B}} W=\widehat{W}^{N}=\mathbb{F}^{\mathbb{N}}$, cf. Thm. 3.14, with the action $(\Delta \circ \widehat{w})(\tau)=$ $\widehat{w}(\tau+N)$ for $\widehat{w} \in \widehat{W}$ and $\tau \in \mathbb{N} N$.

For stabilization we choose a nonempty subset $\mathbb{D}$ of the open unit disc $\{\lambda \in \mathbb{C} ;|\lambda|<1\}$ and, for $\mathbb{F}=\mathbb{R}$ in addition, that $\mathbb{D}$ is stable under conjugation and contains at least one real number, cf. [3, p. 970, (5)]. The saturated submonoid $\mathbb{S}_{\mathbb{D}}$ of all $\mathbb{D}$-stable or just stable polynomials consists of the polynomials in $\mathbf{Z}=\mathbb{F}[\Delta]$ whose roots lie in $\mathbb{D}$. The quotient rings $\mathbf{Z}_{\mathbb{D}}:=\mathbb{F}[\Delta]_{\mathbb{S}_{\mathbb{D}}} \subset \mathbb{F}(\Delta)$, resp. $\mathbf{S}:=\mathbf{S}_{\mathbb{D}}:=\mathbf{Z}_{\mathbb{D}} \cap \mathbb{F}(\Delta)_{\mathrm{pr}}[16$, p. 14] are the rings of stable, resp. of stable and proper rational functions. All these rings are principal ideal domains. If $\Delta-\alpha \in \mathbb{S}_{\mathbb{D}}$ then $\mathbf{Z}_{\mathbb{D}}$ is the quotient ring of $\mathbf{S}_{\mathbb{D}}$ with powers of $(\Delta-\alpha)^{-1}$ as denominators, i.e., $\mathbf{Z}_{\mathbb{D}}=\mathbf{S}_{(\Delta-\alpha)^{-1}}$ [3, (5)]. Algorithms for $\mathbf{S}$ use the fact that this ring is euclidean $[16, \S 2.1]$ or are reduced to standard polynomial algorithms over $\mathbb{F}\left[(\Delta-\alpha)^{-1}\right][3, \S 7]$.

A $\mathbf{Z}$-module $P$ gives rise to its $\mathbf{Z}_{\mathbb{D}}$-quotient module

$$
P_{\mathbb{D}}:=P_{\mathbb{S}_{\mathbb{D}}}:=\left\{s^{-1} x ; x \in P, s \in \mathbb{S}_{\mathbb{D}}\right\} .
$$

In particular, the module $\widehat{W}_{\mathbb{D}}$ is an injective cogenerator over $\mathbf{Z}_{\mathbb{D}}[3, \S 2]$. This was an essential tool in [3] for the construction of compensators and will below be used for periodic systems. All commutative rings above give rise to their $N \times N$-matrix extensions

$$
\begin{array}{ccccc}
\mathbf{Z}=\mathbb{F}[\Delta] & \subset & \mathbf{Z}_{\mathbb{D}}=\mathbf{Z}_{\mathbb{S}_{\mathbb{D}}} & \subset & \mathbf{K}=\mathbb{F}(\Delta) \\
\bigcap_{\mathbf{B}=\mathbf{Z}^{N \times N}} & \subset & \mathbf{B}_{\mathbb{D}}:=\mathbf{B}_{\mathbb{D}_{\mathbb{D}}}=\mathbf{Z}_{\mathbb{D}}^{N \times N} & \subset & \mathbf{Q}=\mathbf{K}^{N \times N} \\
\text { and } \mathbb{F}\left[(\Delta-\alpha)^{-1}\right] \subset \mathbf{S}=\mathbf{S}_{\mathbb{D}} \subset \mathbf{Z}_{\mathbb{D}}, \mathbf{C}:=\mathbf{S}^{N \times N} & \subset \mathbf{B}_{\mathbb{D}}=\mathbf{Z}_{\mathbb{D}}^{N \times N}
\end{array}
$$


to which the theory of Section 4 is applicable. Notice that only constant polynomials in $\mathbf{Z}=\mathbb{F}[\Delta]$ are proper and contained in $\mathbf{S}_{\mathbb{D}}$. By Morita equivalence the signal module $W_{\mathbb{D}}:=W_{\mathbb{S}_{\mathbb{D}}}=\mathbb{F}_{\mathbb{D}}^{\mathbb{N}}$ is an injective cogenerator over $\mathbf{B}_{\mathbb{D}}$.

We assume an IO-behavior with the following data:

$$
\begin{aligned}
& \left(D_{l},-N_{l}\right) \in \mathbf{B}^{p \times(p+m)}=\mathbf{Z}^{N p \times N(p+m)}, D_{l} \in \mathrm{Gl}_{p}(\mathbf{Q})=\mathrm{Gl}_{N p}(\mathbf{K}), \\
& U=\mathbf{B}^{1 \times p}\left(D_{l},-N_{l}\right) \subseteq \mathbf{B}^{1 \times(p+m)}=\mathbf{Z}^{1 \times N(p+m)}, M:=\mathbf{B}^{1 \times(p+m)} / U \\
& M^{0}:=\mathbf{B}^{1 \times p} / \mathbf{B}^{1 \times p} D_{l}, G:=D_{l}^{-1} N_{l} \in \mathbf{Q}^{p \times m}=\mathbf{K}^{N p \times N m}, \\
& \mathcal{B}:=\left\{\left(\begin{array}{c}
y \\
u
\end{array}\right) \in W^{p+m}=\widehat{W}^{N(p+m)} ; D_{l} \circ y=N_{l} \circ u\right\} \cong \operatorname{Hom}_{\mathbf{B}}(M, W) \\
& \mathcal{B}^{0}:=\left\{y \in W^{p}=\widehat{W}^{N p} ; D_{l} \circ y=0\right\} \cong \operatorname{Hom}_{\mathbf{B}}\left(M^{0}, W\right) \\
& \mathcal{B}_{\mathbb{D}}:=\left\{\left(\begin{array}{c}
y \\
u
\end{array}\right) \in W_{\mathbb{D}}^{p+m}=\widehat{W}_{\mathbb{D}}^{N(p+m)} ; D_{l} \circ y=N_{l} \circ u\right\} \cong \operatorname{Hom}_{\mathbf{B}_{\mathbb{D}}}\left(M_{\mathbb{D}}, W_{\mathbb{D}}\right) \\
& \mathcal{B}_{\mathbb{D}}^{0}:=\left\{y \in W_{\mathbb{D}}^{p}=\widehat{W}_{\mathbb{D}}^{N p} ; D_{l} \circ y=0\right\} \cong \operatorname{Hom}_{\mathbf{B}_{\mathbb{D}}}\left(M_{\mathbb{D}}^{0}, W_{\mathbb{D}}\right)
\end{aligned}
$$

where by (61) $\mathcal{B}$ can be interpreted as a (periodic) ${ }_{\mathbf{B}} W$-behavior or as an LTI ${ }_{\mathbf{z}} \widehat{W}$ behavior. In its latter form it admits the standard LTI stabilization theory [7], [16],[4], [3]. It turns out that all LTI results for $\mathcal{B}$ can be translated to results concerning the periodic behavior. We are going to do this below. By definition the IO-behavior is $(\mathbb{D})$ stable if its autonomous part $\mathcal{B}^{0}:=\left\{y \in W^{p}=\widehat{W}^{N p} ; D_{l} \circ y=0\right\}$ has this property. Stability is characterized in the following lemma and requires the characteristic variety and polynomial-exponential signals that we recall for the base field $\mathbb{C}$ and the signal module $\mathbb{C}[\Delta] \mathbb{C}^{\mathbb{N} N}$. Its torsion module admits the primary or modal decomposition

$$
\begin{gathered}
\operatorname{tor}_{\mathbb{C}[\Delta]}\left(\mathbb{C}^{\mathbb{N} N}\right)=\oplus_{\lambda \in \mathbb{C}} \mathbb{C}^{\mathbb{N} N}(\lambda), \mathbb{C}^{\mathbb{N} N}(\lambda)= \begin{cases}\mathbb{C}[\tau / N]\left(\lambda^{\tau / N}\right)_{\tau \in \mathbb{N} N} & \text { if } \lambda \neq 0 \\
\mathbb{C}^{(\mathbb{N} N)} & \text { if } \lambda=0\end{cases} \\
\mathbb{C}^{\mathbb{N} N}(\lambda)=\oplus_{k=0}^{\infty} \mathbb{C} e_{\lambda, k}, e_{\lambda, k}(\tau):= \begin{cases}\left(\begin{array}{c}
\tau / N \\
k
\end{array}\right) \lambda^{(\tau / N)-k} & \text { if } \lambda \neq 0 \\
\delta_{\tau / N, k} & \text { if } \lambda=0\end{cases} \\
(\Delta-\lambda)^{l} \circ e_{\lambda, k}= \begin{cases}e_{\lambda, k-l} & \text { if } k \geq l \\
0 & \text { if } k<l .\end{cases}
\end{gathered}
$$

Here $\mathbb{C}^{(\mathbb{N} N)} \subset \mathbb{C}^{\mathbb{N} N}$ consists of the sequences $\widehat{w}=(\widehat{w}(\tau))_{\tau \in \mathbb{N} N}$ with finite support $\{\tau \in \mathbb{N} N ; \widehat{w}(\tau) \neq 0\}$. The quotients $\tau / N$ come, of course, from the fact that $\mathbb{N} N$ contains multiples of $N$ only and $\mathbb{C}[\tau / N]$ consists of polynomial functions of $\tau / N$. If

$$
\widehat{\mathcal{B}}=\left\{\widehat{w} \in\left(\mathbb{C}^{\mathbb{N} N}\right)^{\ell} ; \widehat{R} \circ \widehat{w}=0\right\}, \widehat{R} \in \mathbb{C}[\Delta]^{k \times \ell}, \operatorname{rank}(\widehat{R})=\ell,
$$

is any autonomous behavior its characteristic variety or set of poles is the finite set

$$
\begin{aligned}
\operatorname{char}(\widehat{\mathcal{B}}): & =\left\{\lambda \in \mathbb{C} ; \operatorname{rank}_{\mathbb{C}}(\widehat{R}(\lambda))<\operatorname{rank}(\widehat{R})=\ell\right\} \\
& =\left\{\lambda \in \mathbb{C} ; \widehat{\mathcal{B}} \bigcap \mathbb{C}^{\mathbb{N} N}(\lambda)^{\ell} \neq 0\right\}=\left\{\lambda \in \mathbb{C} ; \widehat{\mathcal{B}} \bigcap \mathbb{C}^{\ell} e_{\lambda, 0} \neq 0\right\} \text { and then } \\
\widehat{\mathcal{B}} & =\oplus_{\lambda \in \operatorname{char}(\widehat{\mathcal{B}})}\left(\widehat{\mathcal{B}} \bigcap \mathbb{C}^{\mathbb{N} N}(\lambda)^{\ell}\right) \text { (modal decomposition). }
\end{aligned}
$$


Hence all trajectories $\widehat{w}$ of $\widehat{\mathcal{B}}$ are exponentially and asymptotically stable or satisfy $\lim _{\tau \rightarrow \infty} \widehat{w}(\tau)=0$ if and only if $\operatorname{char}(\widehat{\mathcal{B}})$ is contained in the open unit disc. Slightly different statements hold for $\mathbb{F}=\mathbb{R}$. The preceding considerations are applicable to $\mathcal{B}^{0}$ from (78).

Lemma 5.1. ([3, Thm. 3.2]) For the IO-behavior from (78) the following properties are equivalent:

(i) $\mathcal{B}$ is $(\mathbb{D})$-stable, i.e., by definition, $\mathcal{B}_{\mathbb{D}}^{0}=0$ or $M_{\mathbb{D}}^{0}=0$.

(ii) The characteristic variety of $\mathcal{B}^{0}$ is contained in $\mathbb{D}$, i.e.,

$$
\operatorname{char}\left(\mathcal{B}^{0}\right)=\left\{\lambda \in \mathbb{C} ; \operatorname{rank}_{\mathbb{C}}\left(D_{l}(\lambda)\right)<\operatorname{rank}_{\mathbf{Z}}\left(D_{l}\right)=N p\right\} \subset \mathbb{D} .
$$

(iii) $D_{l} \in \mathrm{Gl}_{p}\left(\mathbf{B}_{\mathbb{D}}\right)=\mathrm{Gl}_{N p}\left(\mathbf{Z}_{\mathbb{D}}\right)$.

(iv) (a) $G=D_{l}^{-1} N_{l} \in \mathbf{B}_{\mathbb{D}}^{p \times m}=\mathbf{Z}_{\mathbb{D}}^{N p \times N m}$. (b) $M_{\mathbb{D}}$ is $\mathbf{Z}_{\mathbb{D}}$-free.

According to Cor. 4.5 condition (b) implies that $M_{\mathbb{D}}$ is $\mathbf{B}_{\mathbb{D}}$-free of dimension $m,\left(D_{l},-N_{l}\right)$ has a right inverse in $\mathbf{B}_{\mathbb{D}}^{(p+m) \times p}=\mathbf{Z}_{\mathbb{D}}^{N(p+m) \times N p}$ and that $G=$ $D_{l}^{-1} N_{l}$ is the left coprime factorization of $G$ over $\mathbf{B}_{\mathbb{D}}$.

Since $\mathbb{D}$ is assumed to be a subset of the open unit disc all trajectories of the $(\mathbb{D})$-stable behavior $\mathcal{B}^{0}$ are asymptotically stable and this is the decisive consequence of stability.

Notice that Lemma 5.1 uses the fact that ${ }_{\mathbf{B}_{\mathbb{D}}} W_{\mathbb{D}}$ is an injective cogenerator. A $\mathbb{D}$-stabilizing output feedback controller $\mathcal{C}^{\prime}$ of $\mathcal{B}$ is a behavior that is interconnected to $\mathcal{B}$ in the usual way such that the interconnected behavior $\mathcal{D}^{\prime}$ is a $\mathbb{D}$-stable IO-behavior, i.e., satisfies $\mathcal{D}_{\mathbb{D}}^{\prime 0}=0$; cf. Algorithm 5.2 for the details. This latter condition involves the localized signal space $W_{\mathbb{D}}$ only and therefore it suffices to consider $\mathbf{B}_{\mathbb{D}} W_{\mathbb{D}^{-}}$ behaviors only. We do this in the sequel. Conversely, every such behavior is the localization of a ${ }_{\mathbf{B}} W$-behavior. Following Vidyasagar [16] we construct only $\mathcal{D}^{\prime}$ with proper transfer matrix and call the controllers $\mathcal{C}^{\prime}$ properly $\mathbb{D}$-stabilizing. This requires to use the rings $\mathbf{S}$ and $\mathbf{C}=\mathbf{S}^{N \times N}$ and their modules instead of $\mathbf{Z}_{\mathbb{D}}$ and $\mathbf{B}_{\mathbb{D}}$.

The LTI behavior $\mathcal{B}$ is $\mathbb{D}$-stabilizable, i.e., admits a $\mathbb{D}$-stabilizing compensator, if and only if it satisfies condition (iv),(b), of Lemma 5.1. This means that $G=D_{l}^{-1} N_{l}$ is the left coprime factorization over $\mathbf{B}_{\mathbb{D}}$.

Algorithm 5.2. We assume that the given ${ }_{\mathbf{B}} W$-IO-behavior $\mathcal{B}$ is $\mathbb{D}$-stabilizable or, in other words, that $G=D_{l}^{-1} N_{l}$ is the left coprime factorization over $\mathbf{B}_{\mathbb{D}}$. According to [16, Ch. 5, Thm. 5.2.1], [4], [3, Thm. 3.12] all properly $\mathbb{D}$-stabilizing controllers $\mathcal{C}_{\mathbb{D}}$ of $\mathcal{B}_{\mathbb{D}}$ and their interconnected behaviors $\mathcal{D}_{\mathbb{D}}$ are obtained with the following steps: We apply Lemma 4.8 and Lemma 4.7 to the rings $\mathbf{S} \subset \mathbf{K}=\operatorname{quot}(\mathbf{S})$ and $\mathbf{C}=\mathbf{S}^{N \times N} \subset$ $\mathbf{Q}=\mathbf{K}^{N \times N}$ and the transfer matrix $G=D_{l}^{-1} N_{l}$ and construct the matrices

$$
D_{l}^{0}, N_{l}^{0}, D_{r}^{0}, N_{r}^{0}, S_{l}^{0}, R_{l}^{0}, S_{r}^{0}, R_{r}^{0} \in \mathbf{C}^{\bullet \times \bullet}=\mathbf{S}^{N \bullet \times N \bullet}
$$

such that (i), (ii), (iii) of Lemma 4.7 hold. In particular, $G=\left(D_{l}^{0}\right)^{-1} N_{l}^{0}=N_{r}^{0}\left(D_{r}^{0}\right)^{-1}$ are the left, resp. right coprime factorizations over $\mathbf{S}$ and over $\mathbf{C}$. All other quadrupels $S_{l}, R_{l}, S_{r}, R_{r}$ with the same properties are obtained by the choice of an arbitrary matrix $X \in \mathbf{C}^{m \times p}=\mathbf{S}^{N m \times N p}$ and

$$
\left(\begin{array}{c}
S_{r} \\
-R_{r}
\end{array}\right)=\left(\begin{array}{c}
S_{r}^{0} \\
-R_{r}^{0}
\end{array}\right)-\left(\begin{array}{c}
N_{r}^{0} \\
D_{r}^{0}
\end{array}\right) X,\left(R_{l}, S_{l}\right)=\left(R_{l}^{0}, S_{l}^{0}\right)+X\left(D_{l}^{0},-N_{l}^{0}\right) .
$$


They satisfy

$$
\left(\begin{array}{cc}
D_{l}^{0} & -N_{l}^{0} \\
R_{l} & S_{l}
\end{array}\right) \in \mathrm{Gl}_{p+m}(\mathbf{C})=\mathrm{Gl}_{N(p+m)}(\mathbf{S}),\left(\begin{array}{cc}
D_{l}^{0} & -N_{l}^{0} \\
R_{l} & S_{l}
\end{array}\right)^{-1}=\left(\begin{array}{cc}
S_{r} & N_{r}^{0} \\
-R_{r} & D_{r}^{0}
\end{array}\right) .
$$

For almost all $X$ the matrices $S_{l}$ and $S_{r}$ satisfy $\operatorname{det}_{\mathbf{S}}\left(S_{l}\right) \neq 0$ and $\operatorname{det}_{\mathbf{S}}\left(S_{r}\right) \neq 0$ or, equivalently, $S_{l} \in \mathrm{Gl}_{m}(\mathbf{Q})=\mathrm{Gl}_{N m}(\mathbf{K})$ and $S_{r} \in \mathrm{Gl}_{p}(\mathbf{Q})=\mathrm{Gl}_{N p}(\mathbf{K})$. The left coprime factorization $G=\left(D_{l}^{0}\right)^{-1} N_{l}^{0}$ over $\mathbf{S}$ and $\mathbf{C}$ is also such over $\mathbf{Z}_{\mathbb{D}} \supset \mathbf{S}$ and $\mathbf{B}_{\mathbb{D}} \supset \mathbf{C}$. According to Lemma 4.7 it is unique up to row equivalence and therefore

$$
\begin{gathered}
G=D_{l}^{-1} N_{l}=\left(D_{l}^{0}\right)^{-1} N_{l}^{0}, \mathbf{B}_{\mathbb{D}}^{1 \times p}\left(D_{l},-N_{l}\right)=\mathbf{B}_{\mathbb{D}}^{1 \times p}\left(D_{l}^{0},-N_{l}^{0}\right) \Longrightarrow \\
\mathcal{B}_{\mathbb{D}}=\left\{\left(\begin{array}{c}
y_{1} \\
u_{1}
\end{array}\right) \in W_{\mathbb{D}}^{p+m} ; D_{l} \circ y_{1}=N_{l} \circ u_{1}\right\}=\left\{\left(\begin{array}{c}
y_{1} \\
u_{1}
\end{array}\right) \in W_{\mathbb{D}}^{p+m} ; D_{l}^{0} \circ y_{1}=N_{l}^{0} \circ u_{1}\right\} .
\end{gathered}
$$

The controller $\mathcal{C}_{\mathbb{D}}$ of $\mathcal{B}_{\mathbb{D}}$ as $\mathbf{B}_{\mathbb{D}} W_{\mathbb{D}}$-behavior is given by the equations

$$
\mathcal{C}_{\mathbb{D}}:=\left\{\left(\begin{array}{l}
u_{2} \\
y_{2}
\end{array}\right) \in W_{\mathbb{D}}^{p+m} ; R_{l} \circ u_{2}+S_{l} \circ y_{2}=0\right\} .
$$

If $S_{l} \in \mathrm{Gl}_{m}(\mathbf{Q})$ the controller is also an IO-behavior with input $u_{2}$ and output $y_{2}$. The output feedback ${ }_{\mathbf{B}_{\mathbb{D}}} W_{\mathbb{D}}$-behavior $\mathcal{D}_{\mathbb{D}}$ is defined by (see Figure 1)

$$
\begin{aligned}
& \begin{aligned}
&\left.\left.\begin{array}{rl}
u & := \\
\mathcal{D}_{\mathbb{D}} & :=\left\{\begin{array}{l}
u_{2} \\
u_{1}
\end{array}\right), y:=\left(\begin{array}{c}
y_{1} \\
y_{2}
\end{array}\right) \in W_{\mathbb{D}}^{p+m} \\
u
\end{array}\right) \in W_{\mathbb{D}}^{(p+m)+(p+m)} ;\left(\begin{array}{c}
y_{1} \\
u_{1}+y_{2}
\end{array}\right) \in \mathcal{B}_{\mathbb{D}},\left(\begin{array}{c}
y_{2} \\
u_{2}+y_{1}
\end{array}\right) \in \mathcal{C}_{\mathbb{D}}\right\} \\
&=\left\{\left(\begin{array}{l}
y \\
u
\end{array}\right) \in W_{\mathbb{D}}^{(p+m)+(p+m)} ;\left\{\begin{array}{l}
D_{l}^{0} \circ y_{1}=N_{l}^{0} \circ\left(u_{1}+y_{2}\right) \\
R_{l} \circ\left(u_{2}+y_{1}\right)+S_{l} \circ y_{2}=0
\end{array}\right\}\right. \\
&=\left\{\left(\begin{array}{c}
y \\
u
\end{array}\right) \in W_{\mathbb{D}}^{(p+m)+(p+m)} ; D \circ y=N \circ u\right\}
\end{aligned} \\
& \text { where } D:=\left(\begin{array}{cc}
D_{l}^{0} & -N_{l}^{0} \\
R_{l} & S_{l}
\end{array}\right) \in \mathrm{Gl}_{p+m}(\mathbf{C}), N:=\left(\begin{array}{cc}
0 & N_{l}^{0} \\
-R_{l} & 0
\end{array}\right) \in \mathbf{C}^{(p+m) \times(p+m)} .
\end{aligned}
$$

The numbering $u=\left(\begin{array}{l}u_{2} \\ u_{1}\end{array}\right)$ of the components of $u$ is chosen such that both $u$ and $y$ belong to $W_{\mathbb{D}}^{p+m}$. Equation 85 implies that $\mathcal{D}_{\mathbb{D}}$ is a $\mathbf{B}_{\mathbb{D}} W_{\mathbb{D}}$ IO-behavior with input $u$ and

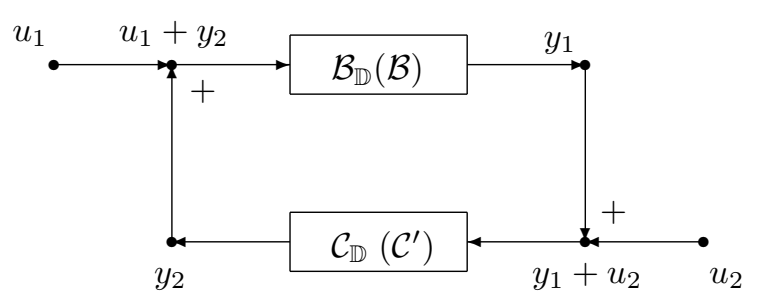

Figure 1: The interconnected behavior $\mathcal{D}_{\mathbb{D}}\left(\mathcal{D}^{\prime}\right)$.

output $y$ and is $\mathbb{D}$-stable, cf. Lemmas 4.4 and 5.1. Its transfer matrix in $\mathbf{C}^{(p+m) \times(p+m)}$ is

$$
H=\left(\begin{array}{cc}
H_{y_{1}, u_{2}} & H_{y_{1}, u_{1}} \\
H_{y_{2}, u_{2}} & H_{y_{2}, u_{1}}
\end{array}\right):=D^{-1} N=\left(\begin{array}{cc}
S_{r} & N_{r}^{0} \\
-R_{r} & D_{r}^{0}
\end{array}\right)\left(\begin{array}{cc}
0 & N_{l}^{0} \\
-R_{l} & 0
\end{array}\right) \underset{(85)}{=}\left(\begin{array}{cc}
-N_{r}^{0} R_{l} & S_{r} N_{l}^{0} \\
-D_{r}^{0} R_{l} & -R_{r} N_{l}^{0}
\end{array}\right) .
$$


The unique controllable ${ }_{\mathrm{B}} W$-compensator $\mathcal{C}^{\prime}$ of $\mathcal{B}$ with localization $\mathcal{C}_{\mathbb{D}}=\mathcal{C}_{\mathbb{D}}^{\prime}$ is obtained as follows: Define the f.g. projective modules

$$
\begin{aligned}
& V:=\mathbf{B}_{\mathbb{D}}^{1 \times m}\left(R_{l}, S_{l}\right) \subseteq \mathbf{B}_{\mathbb{D}}^{1 \times(p+m)}, V^{\prime}:=\mathbf{B}^{1 \times(p+m)} \bigcap V \subseteq \mathbf{B}^{1 \times(p+m)} \\
& \Longrightarrow \mathbf{B}^{1 \times(p+m)} / V^{\prime} \subseteq \mathbf{B}_{\mathbb{D}}^{1 \times(p+m)} / V, V_{\mathbb{D}}^{\prime}=V, \\
& \Longrightarrow \operatorname{dim}_{\mathbf{Z}}\left(V^{\prime}\right)=\operatorname{dim}_{\mathbf{Z}_{\mathbb{D}}}\left(V_{\mathbb{D}}^{\prime}\right)=\operatorname{dim}_{\mathbf{Z}_{\mathbb{D}}}(V)=N^{2} \operatorname{dim}_{\mathbf{B}_{\mathbb{D}}}(V)=N^{2} m .
\end{aligned}
$$

Moreover, since $\mathbf{B}_{\mathbb{D}}^{1 \times(p+m)} / V$ is $\mathbf{Z}$-free so is its $\mathbf{Z}$-submodule $\mathbf{B}^{1 \times(p+m)} / V^{\prime}$ and thus the latter is B-projective. Again by Lemma 4.2 we infer that $V^{\prime}$, resp. $\mathbf{B}^{1 \times(p+m)} / V^{\prime}$ are $\mathbf{B}$-free of dimensions $m$, resp. $p$. In particular, $V=\mathbf{B}^{1 \times m}\left(R_{l}^{\prime}, S_{l}^{\prime}\right)$ where $\left(R_{l}^{\prime}, S_{l}^{\prime}\right) \in$ $\mathbf{B}^{m \times(p+m)}$ has B-linearly independent rows. Define

$$
\begin{aligned}
& \mathcal{C}^{\prime}:=V^{\prime \perp}:=\left\{\left(\begin{array}{l}
u_{2} \\
y_{2}
\end{array}\right) \in W^{p+m} ; R_{l}^{\prime} \circ u_{2}+S_{l}^{\prime} \circ y_{2}=0\right\} . \\
& V_{\mathbb{D}}^{\prime}=\mathbf{B}_{\mathbb{D}}^{1 \times m}\left(R_{l}^{\prime}, S_{l}^{\prime}\right)=V=\mathbf{B}_{\mathbb{D}}^{1 \times m}\left(R_{l}, S_{l}\right) \\
& \Longrightarrow \mathcal{C}_{\mathbb{D}}^{\prime}=\left(V^{\prime \perp}\right)_{\mathbb{D}}=V^{\perp}=\mathcal{C}_{\mathbb{D}} .
\end{aligned}
$$

Therefore $\mathcal{C}_{\mathbb{D}}$ is the localization of $\mathcal{C}^{\prime}$. Since $\mathbf{B}^{1 \times(p+m)} / V^{\prime}$ is $\mathbf{B}$-free the compensator $\mathcal{C}^{\prime}$ is controllable. The algorithmic computation of $V^{\prime}$ and $\mathcal{C}^{\prime}$ is explained in [3, §7].

The behavior $\mathcal{C}^{\prime}$ is the unique controllable one with $\mathcal{C}_{\mathbb{D}}^{\prime}=\mathcal{C}_{\mathbb{D}}$. There are less useful noncontrollable behaviors $\mathcal{C}^{\prime \prime}$ with $\mathcal{C}_{\mathbb{D}}^{\prime \prime}=\mathcal{C}_{\mathbb{D}}$.

The interconnection of the given behavior $\mathcal{B}$ with $\mathcal{C}^{\prime}$ is given by

$$
\begin{aligned}
& \mathcal{D}^{\prime}=\left\{\left(\begin{array}{c}
y \\
u
\end{array}\right) \in W^{(p+m)+(p+m)} ; D^{\prime} \circ y=N^{\prime} \circ u\right\} \text { where } \\
& D^{\prime}:=\left(\begin{array}{cc}
D_{l} & -N_{l} \\
R_{l}^{\prime} & S_{l}^{\prime}
\end{array}\right) \in \mathbf{B}^{(p+m) \times(p+m)} \bigcap \mathrm{Gl}_{p+m}(\mathbf{Q}) \\
& N^{\prime}:=\left(\begin{array}{cc}
0 & N_{l} \\
-R_{l}^{\prime} & 0
\end{array}\right) \in \mathbf{B}^{(p+m) \times(p+m)} .
\end{aligned}
$$

Since $\mathcal{B}_{\mathbb{D}}$ is the localization of $\mathcal{B}$ and $\mathcal{C}_{\mathbb{D}}$ that of $\mathcal{C}^{\prime}$ we infer (cf. [3, Cor. 3.8])

$$
\begin{aligned}
\mathbf{B}_{\mathbb{D}}^{1 \times(p+m)}\left(D^{\prime},-N^{\prime}\right) & =\mathbf{B}_{\mathbb{D}}^{1 \times(p+m)}(D,-N) \\
\Longrightarrow \mathcal{D}_{\mathbb{D}}^{\prime}=\mathcal{D}_{\mathbb{D}}, \mathcal{D}^{\prime 0} & =\mathcal{D}_{\mathbb{D}}^{0}=0, H=D^{\prime-1} N^{\prime}=D^{-1} N .
\end{aligned}
$$

Summing up we obtain

Theorem 5.3. Let $\mathcal{B}$ be a $\mathbb{D}$-stabilizable periodic IO-behavior, i.e., $\left(D_{l},-N_{l}\right)$ has a right inverse in $\mathbf{B}_{\mathbb{D}}^{(p+m) \times p}$. The behaviors $\mathcal{C}^{\prime}, \mathcal{D}^{\prime}$, constructed above, are ${ }_{\mathrm{B}} W$ behaviors, i.e. periodic behaviors. The feedback interconnection $\mathcal{D}^{\prime}$ is a $\mathbb{D}$-stable IO-behavior with proper transfer matrix $H \in \mathbf{C}^{(p+m) \times(p+m)}, \mathbf{C}=\mathbf{S}^{N \times N}$, from (89). Thus the compensator $\mathcal{C}^{\prime}$ is properly $\mathbb{D}$-stabilizing and moreover controllable and all such compensators are obtained in the described fashion. For almost all $X \in \mathbf{C}^{m \times p}$ from (84) the matrices $S_{l}$ and $S_{l}^{\prime}$ belong to $\mathrm{Gl}_{m}(\mathbf{Q})$ and both $\mathcal{C}_{\mathbb{D}}$ and $\mathcal{C}^{\prime}$ are IObehaviors with input $u_{2}$ and output $y_{2}$.

Remark 5.4. (Properness of the controller) Consider the data of Algorithm 5.2 and Thm. 5.3. By construction the interconnected IO behaviors $\mathcal{D}^{\prime}$ and $\mathcal{D}_{\mathbb{D}}$ have a proper transfer matrix $H$ whereas properness of the plant transfer matrix $G=D_{l}^{-1} N_{l}=$ 
$\left(D_{l}^{0}\right)^{-1} N_{l}^{0}$ is not assumed. Recall that almost all constructed controllers are IO behaviors and thus have a transfer matrix $G_{\mathcal{C}}$, in detail

$$
\begin{aligned}
& \mathcal{C}^{\prime}=\left\{\left(\begin{array}{l}
u_{2} \\
y_{2}
\end{array}\right) \in W^{p+m} ; S_{l}^{\prime} \circ y_{2}=-R_{l}^{\prime} \circ u_{2}\right\}, \\
& \mathcal{C}_{\mathbb{D}}^{\prime}=\mathcal{C}_{\mathbb{D}}=\left\{\left(\begin{array}{l}
u_{2} \\
y_{2}
\end{array}\right) \in W_{\mathbb{D}}^{p+m} ; S_{l} \circ y_{2}=-R_{l} \circ u_{2}\right\} \\
& S_{l}^{\prime} \in \mathbf{B}^{m \times m} \bigcap \mathrm{Gl}_{m}(\mathbf{Q}), S_{l} \in \mathbf{C}^{m \times m} \bigcap \mathrm{Gl}_{m}(\mathbf{Q}), R_{l}^{\prime} \in \mathbf{B}^{m \times p}, R_{l} \in \mathbf{C}^{m \times p}, \\
& G_{\mathcal{C}}=-\left(S_{l}^{\prime}\right)^{-1} R_{l}^{\prime}=-S_{l}^{-1} R_{l} \in \mathbf{Q}^{m \times p}=\mathbb{F}(\Delta)^{N m \times N p} .
\end{aligned}
$$

Properness of $G_{\mathcal{C}}$, i.e., $G_{\mathcal{C}} \in \mathbb{F}(\Delta)_{\mathrm{pr}}^{N m \times N p}$, is necessary and sufficient in order that $\mathcal{C}^{\prime}$ can be implemented with elementary building blocks. In [3, Thm. 3.27] it was shown that almost all controllers from Thm. 5.3 are IO behaviors with a proper transfer matrix $G_{\mathcal{C}}$. Moreover, if the transfer matrix $G$ of the plant is strictly proper then all controllers $\mathcal{C}^{\prime}$ from Thm. 5.3 are IO behaviors with proper transfer matrix, cf. [16, Cor. 5.2.20]. Symmetrically, if the controller is an IO behavior with strictly proper transfer matrix then the plant transfer matrix $G$ is proper.

\section{Tracking and disturbance rejection}

We assume a $\mathbb{D}$-stabilizable plant as in Theorem 5.3 and consider the properly $\mathbb{D}$ stabilizing controllers $\mathcal{C}^{\prime}$ and $\mathcal{C}_{\mathbb{D}}^{\prime}=\mathcal{C}_{\mathbb{D}}$ of this theorem. The input signals $u_{1}$, resp. $u_{2}$ of $\mathcal{D}^{\prime}$ are interpreted as disturbances of the input, resp. of the output of $\mathcal{B}$. In addition we assume a reference signal $r \in W^{p}$. We assume that a nonzero $\psi \in \mathbb{F}[\Delta]$ is given such that

$$
\psi \circ r=0, \psi \circ u_{1}=0, \psi \circ u_{2}=0,
$$

i.e., that the signals $u_{1}, u_{2}, r$ are generated by an autonomous system. We consider the interconnected tracking system (see Figure 2)

$$
\begin{aligned}
& \mathcal{T}^{\prime}:=\left\{\left(\begin{array}{l}
y \\
u \\
r
\end{array}\right) \in W^{(p+m)+(p+m)+p} ;(*)\right\} \text { where } \\
& (*) D_{l} \circ y_{1}=N_{l} \circ\left(u_{1}+y_{2}\right), S_{l}^{\prime} \circ y_{2}+R_{l}^{\prime} \circ\left(u_{2}+y_{1}-r\right)=0 \\
& \psi \circ u_{1}=0, \psi \circ u_{2}=0, \psi \circ r=0 .
\end{aligned}
$$

So the input signal of the controller is the error signal $e:=y_{1}+u_{2}-r$ that is the

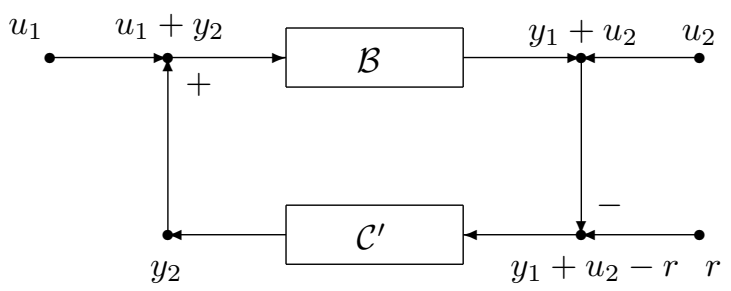

Figure 2: The tracking behavior $\mathcal{T}^{\prime}$. 
difference between the disturbed output $y_{1}+u_{2}$ of the plant and the reference signal $r$. The aim is to construct controllers with $\mathbb{D}$-stable $e$ for all $u_{1}, u_{2}, r$ satisfying (95). The error behavior is the behavior of all error signals, i.e.,

$$
\begin{aligned}
\mathcal{E}^{\prime} & :=\operatorname{im}\left(\mathcal{T}^{\prime} \rightarrow W^{p},\left(\begin{array}{l}
y \\
u \\
r
\end{array}\right) \mapsto e=y_{1}+u_{2}-r\right) \\
& =\left\{y_{1}+u_{2}-r \in W^{p} ;\left(\begin{array}{l}
y \\
u \\
r
\end{array}\right) \in \mathcal{T}^{\prime}\right\} .
\end{aligned}
$$

The controller $\mathcal{C}^{\prime}$ is said to track the reference signal $r$ and to reject disturbances $u_{1}$ and $u_{2}$ that satisfy $(95)$ if $\mathcal{E}_{\mathbb{D}}^{\prime}=0$. If this is the case all error signals are asymptotically stable, cf. (79)-(81) and Lemma 5.1.

Theorem 6.1. (i) The $\mathbb{D}$-stabilizing controller $\mathcal{C}^{\prime}$ from Thm. 5.3 tracks the reference signal $r$ and rejects the disturbances $u_{1}$ and $u_{2}$ satisfying (95) if and only if

$$
Z:=\psi^{-1} S_{r} \in \mathbf{B}_{\mathbb{D}}^{p \times p} .
$$

(ii) There is such a controller if and only if the inhomogeneous linear matrix equation

$$
S_{r}^{0}=N_{r}^{0} X+\psi Z
$$

has a solution $X \in \mathbf{C}^{m \times p}=\mathbf{S}^{N m \times N p}$ and $Z \in \mathbf{B}_{\mathbb{D}}^{p \times(p+m)}$.

The computation of the solution $(X, Z)$ is described in $[3, \S 7]$.

Proof. (i) Since the functor $(-)_{\mathbb{D}}$ is exact and hence

$$
\mathcal{E}_{\mathbb{D}}^{\prime}:=\operatorname{im}\left(\mathcal{T}_{\mathbb{D}}^{\prime} \rightarrow W_{\mathbb{D}}^{p},\left(\begin{array}{c}
y \\
u \\
r
\end{array}\right) \mapsto e=y_{1}+u_{2}-r\right)
$$

the condition $\mathcal{E}_{\mathbb{D}}^{\prime}=0$ holds if and only if the following implication holds:

$$
\left\{\begin{array}{l}
\left(\begin{array}{l}
y \\
u \\
r
\end{array}\right) \in W_{\mathbb{D}}^{(p+m)+(p+m)+p} \\
D_{l} \circ y_{1}=N_{l} \circ\left(u_{1}+y_{2}\right), S_{l}^{\prime} \circ y_{2}+R_{l}^{\prime} \circ\left(u_{2}+y_{1}-r\right)=0 \Longrightarrow e=0 \\
\psi \circ u_{1}=0, \psi \circ u_{2}=0, \psi \circ r=0
\end{array}\right.
$$

Since

$$
\mathbf{B}_{\mathbb{D}}^{1 \times p}\left(D_{l},-N_{l}\right)=\mathbf{B}_{\mathbb{D}}^{1 \times p}\left(D_{l}^{0},-N_{l}^{0}\right), \mathbf{B}_{\mathbb{D}}^{1 \times m}\left(R_{l}^{\prime}, S_{l}^{\prime}\right)=\mathbf{B}_{\mathbb{D}}^{1 \times m}\left(R_{l}, S_{l}\right)
$$

the implication (100) is equivalent to the implication

$$
\left\{\begin{array}{l}
\left(\begin{array}{l}
y \\
u \\
r
\end{array}\right) \in W_{\mathbb{D}}^{(p+m)+(p+m)+p}, y=\left(\begin{array}{l}
y_{1} \\
y_{2}
\end{array}\right), u=\left(\begin{array}{l}
u_{2} \\
u_{1}
\end{array}\right), \\
D_{l}^{0} \circ y_{1}=N_{l}^{0} \circ\left(u_{1}+y_{2}\right), S_{l} \circ y_{2}+R_{l} \circ\left(u_{2}+y_{1}-r\right)=0 \Longrightarrow e=0 \\
\psi \circ u_{1}=0, \psi \circ u_{2}=0, \psi \circ r=0
\end{array}\right.
$$

or, in shorter notation with (88), to

$$
\left\{\begin{array}{l}
D \circ\left(\begin{array}{l}
y_{1} \\
y_{2}
\end{array}\right)=N \circ\left(\begin{array}{c}
u_{2}-r \\
u_{1}
\end{array}\right) \text { or } y=H \circ\left(\begin{array}{c}
u_{2}-r \\
u_{1}
\end{array}\right) \\
\psi \circ\left(\begin{array}{l}
u \\
r
\end{array}\right)=0
\end{array} \Longrightarrow e=0 .\right.
$$


With $H=\left(\begin{array}{cc}-N_{r}^{0} R_{l} & S_{r} N_{l}^{0} \\ -D_{r}^{0} R_{l} & -R_{r} N_{l}^{0}\end{array}\right)$ we get the equivalent implication

$$
\begin{gathered}
\psi \circ\left(\begin{array}{l}
u \\
r
\end{array}\right)=0 \Longrightarrow e=y_{1}+u_{2}-r=\left(\operatorname{id}_{p}-N_{r}^{0} R_{l}\right) \circ\left(u_{2}-r\right)+S_{r} N_{l}^{0} \circ u_{1} \\
\underset{(105)}{=} S_{r} D_{l}^{0} \circ\left(u_{2}-r\right)+S_{r} N_{l}^{0} \circ u_{1}=\left(S_{r} D_{l}^{0}, S_{r} N_{l}^{0},-S_{r} D_{l}^{0}\right) \circ\left(\begin{array}{c}
u \\
r
\end{array}\right)=0 .
\end{gathered}
$$

Here we used (85), i.e.,

$$
\left(\begin{array}{cc}
S_{r} & N_{r}^{0} \\
-R_{r} & D_{r}^{0}
\end{array}\right)\left(\begin{array}{cc}
D_{l}^{0} & -N_{l}^{0} \\
R_{l} & S_{l}
\end{array}\right)=\left(\begin{array}{cc}
\operatorname{id}_{p} & 0 \\
0 & \operatorname{id}_{m}
\end{array}\right) \Longrightarrow \mathrm{id}_{p}-N_{r}^{0} R_{l}=S_{r} D_{l}^{0} .
$$

We finally derive the equivalent implication

$$
\forall\left(\begin{array}{c}
u_{2} \\
u_{1} \\
r
\end{array}\right) \in W_{\mathbb{D}}^{p+m+p}: \psi \circ\left(\begin{array}{c}
u_{2} \\
u_{1} \\
r
\end{array}\right)=0 \Longrightarrow\left(S_{r} D_{l}^{0}, S_{r} N_{l}^{0},-S_{r} D_{l}^{0}\right) \circ\left(\begin{array}{c}
u_{2} \\
u_{1} \\
r
\end{array}\right)=0 .
$$

Since ${ }_{\mathbf{B}_{\mathbb{D}}} W_{\mathbb{D}}$ is an injective cogenerator this is equivalent to

$$
\begin{aligned}
& \left(S_{r} D_{l}^{0}, S_{r} N_{l}^{0},-S_{r} D_{l}^{0}\right) \in \mathbf{B}_{\mathbb{D}}^{p \times(p+m+p)} \psi \\
& \Longleftrightarrow \psi^{-1} S_{r}\left(D_{l}^{0},-N_{l}^{0}\right) \in \mathbf{B}_{\mathbb{D}}^{p \times(p+m)} \\
& \Longleftrightarrow Z:=\psi^{-1} S_{r}=\psi^{-1} S_{r}\left(D_{l}^{0},-N_{l}^{0}\right)\left(\begin{array}{c}
S_{r} \\
-R_{r}
\end{array}\right) \in \mathbf{B}_{\mathbb{D}}^{p \times p} .
\end{aligned}
$$

(ii) Recall from (84) that

$$
\left(\begin{array}{c}
S_{r} \\
-R_{r}
\end{array}\right)=\left(\begin{array}{c}
S_{r}^{0} \\
-R_{r}^{0}
\end{array}\right)-\left(\begin{array}{c}
N_{r}^{0} \\
D_{r}^{0}
\end{array}\right) X
$$

Inserting this into (98) furnishes the inhomogeneous equation (99)

$$
S_{r}^{0}=N_{r}^{0} X+\psi Z \text {. }
$$

So (99) follows from the properties of the controller $\mathcal{C}^{\prime}$. If, conversely, (99) has a solution $(X, Z)$ one uses Algorithm 5.2 to define the controller $\mathcal{C}_{\mathbb{D}}$ and then $\mathcal{C}^{\prime}$ with this $X$. Then (99) implies (98) and therefore the controller $\mathcal{C}^{\prime}$ tracks $r$ and rejects $u_{1}$ and $u_{2}$.

A more general tracking interconnection $\mathcal{T}^{\prime}$ than in (96) assumes an additional $\mathbb{D}$-stable IO-behavior $\mathcal{B}_{2}$ with proper (and $\mathbb{D}$-stable) transfer matrix $T_{l} \in \mathbf{C}^{m \times p}$ (cf. Lemma 5.1) that transforms the reference signal $r$ of dimension $p$ into its output $r_{2}$ of dimension $m$ :

$$
\begin{gathered}
\mathcal{B}_{2}:=\left\{\left(\begin{array}{c}
r_{2} \\
r
\end{array}\right) \in W^{m+p} ; D_{l}^{2} \circ r_{2}=N_{l}^{2} \circ r\right\}, \\
\left(D_{l}^{2},-N_{l}^{2}\right) \in \mathbf{B}^{m \times(m+p)}, D_{l}^{2} \in \mathrm{Gl}_{m}(\mathbf{Q}), T_{l}:=\left(D_{l}^{2}\right)^{-1} N_{l}^{2} \in \mathbf{C}^{p+m} .
\end{gathered}
$$

Notice that $\mathcal{B}_{2}$ can be implemented since $T_{l}$ is proper. From Lemma 5.1 we know that $D_{l}^{2} \in \mathrm{Gl}_{m}\left(\mathbf{B}_{\mathbb{D}}\right)$ and that $T_{l}=\left(D_{l}^{2}\right)^{-1} N_{l}^{2}$ is a left coprime factorization over $\mathbf{B}_{\mathbb{D}}$. For a given controller $\mathcal{C}^{\prime}$ according to Thm. 5.3 the generalized interconnected tracking behavior $\mathcal{T}^{\prime}$ is defined by the equations

$$
\begin{aligned}
& D_{l} \circ y_{1}=N_{l} \circ\left(u_{1}+y_{2}\right), S_{l}^{\prime} \circ y_{2}+R_{l}^{\prime} \circ\left(y_{1}+u_{2}\right)=r_{2}, D_{l}^{2} \circ r_{2}=N_{l}^{2} \circ r \\
& \psi \circ r=0, \psi \circ u_{1}=0, \psi \circ u_{2}=0 .
\end{aligned}
$$

The error signal is $e:=y_{1}+u_{2}-r$ again. By definition the matrices $\left(R_{l}^{\prime}, S_{l}^{\prime}, T_{l}\right)$ form an $(R, S, T)$-controller if all error signals $e$ of $\mathcal{T}^{\prime}$ are $\mathbb{D}$-stable, i.e., if the (autonomous) error behavior $\mathcal{E}$ of all error signals is $\mathbb{D}$-stable or satisfies $\mathcal{E}_{\mathbb{D}}=0$. 
Theorem 6.2. Consider the $\mathbb{D}$-stable IO-behavior $\mathcal{B}_{2}$ with proper transfer matrix $T_{l}$ from (109) and a stabilizing controller $\mathcal{C}^{\prime}$ according to Thm. 5.3 with its associated data. The matrices $\left(R_{l}^{\prime}, S_{l}^{\prime}, T_{l}\right)$ form an $(R, S, T)$-controller if and only if

$$
\psi^{-1} S_{r}, \psi^{-1} N_{r}^{0}\left(T_{l}-R_{l}\right) \in \mathbf{B}_{\mathbb{D}}^{p \times p} .
$$

Proof. For signals with components in $W_{\mathbb{D}}$ the equations (110) are equivalent to

$$
\begin{aligned}
& D_{l}^{0} \circ y_{1}=N_{l}^{0} \circ\left(u_{1}+y_{2}\right), S_{l} \circ y_{2}+R_{l} \circ\left(y_{1}+u_{2}\right)=r_{2}, D_{l}^{2} \circ r_{2}=N_{l}^{2} \circ r, \\
& \psi \circ r=0, \psi \circ u_{1}=0, \psi \circ u_{2}=0, y, u, r, r_{2} \in W_{\mathbb{D}}^{\bullet} .
\end{aligned}
$$

Due to

$$
\begin{gathered}
\left(\begin{array}{cc}
D_{l}^{0} & -N_{l}^{0} \\
R_{l} & S_{l}
\end{array}\right)^{-1}=\left(\begin{array}{cc}
S_{r} & N_{r}^{0} \\
-R_{r} & D_{r}^{0}
\end{array}\right) \in \mathrm{Gl}_{p+m}(\mathbf{C}), D_{l}^{2} \in \mathrm{Gl}_{m}\left(\mathbf{B}_{\mathbb{D}}\right), T_{l} \in \mathbf{C}^{m \times p}, \\
r_{2}=T_{l} \circ r, \mathrm{id}_{p}=S_{r} D_{l}^{0}+N_{r}^{0} R_{l}, H=\left(\begin{array}{cc}
-N_{r}^{0} R_{l} & S_{r} N_{l}^{0} \\
-D_{r}^{0} R_{l} & -R_{r} N_{l}^{0}
\end{array}\right)
\end{gathered}
$$

the equations (112) are equivalent to

$$
y=H \circ u+\left(\begin{array}{cc}
S_{r} & N_{r}^{0} \\
-R_{r} & D_{r}^{0}
\end{array}\right)\left(\begin{array}{c}
0 \\
T_{l}
\end{array}\right) \circ r, \psi \circ r=0, \psi \circ u_{1}=0, \psi \circ u_{2}=0
$$

that imply

$$
\begin{aligned}
& y_{1}=-N_{r}^{0} R_{l} \circ u_{2}+S_{r} N_{l}^{0} \circ u_{1}+N_{r}^{0} T_{l} \circ r, \\
& e=y_{1}+u_{2}-r=\left(S_{r} D_{l}^{0}, S_{r} N_{l}^{0}, N_{r}^{0} T_{l}-\mathrm{id}_{p}\right) \circ\left(u_{2}, u_{1}, r\right)^{\top}, \\
& N_{r}^{0} T_{l}-\mathrm{id}_{p}=N_{r}^{0}\left(T_{l}-R_{l}\right)-S_{r} D_{l}^{0} .
\end{aligned}
$$

By definition the matrices $\left(R_{l}^{\prime}, S_{l}^{\prime}, T_{l}\right)$ define an $(R, S, T)$-controller if and only if the equations (112) imply $e=0$. By means of (115) this is equivalent to the implication

$$
\psi \circ\left(u_{2}, u_{1}, r\right)^{\top}=0 \Longrightarrow\left(S_{r} D_{l}^{0}, S_{r} N_{l}^{0}, N_{r}^{0} T_{l}-\mathrm{id}_{p}\right) \circ\left(u_{2}, u_{1}, r\right)^{\top}=0 .
$$

By the same argument as in the proof of Thm. 6.1 equations (116) and (112) are equivalent and this completes the proof.

Remark 6.3. In Thm. 6.1 assume that $S_{r}=\psi S_{r}^{\prime}, S_{r}^{\prime} \in \mathbf{B}_{\mathbb{D}}^{p \times p}$. Then condition (98) is trivially satisfied and moreover

$$
\operatorname{id}_{p}=D_{l}^{0} S_{r}+N_{l}^{0} R_{r}=\left(\psi D_{l}^{0}\right) S_{r}^{\prime}+N_{l}^{0} R_{r}
$$

This implies that $\left(\psi D_{l}^{0},-N_{l}^{0}\right)$ is right invertible over $\mathbf{B}_{\mathbb{D}}$. Notice that in (98) and (99) $\psi$ can be multiplied with a unit in $\mathbf{Z}_{\mathbb{D}}=\mathbf{S}_{(\Delta-\alpha)^{-1}}$ and hence we may assume that $\psi \in \mathbf{S}$.

In the sequel we assume that $\psi \in \mathbf{S}$ and that $\left(\psi D_{l}^{0},-N_{l}^{0}\right)$ has a right inverse $\left(\begin{array}{c}S_{r}^{\prime} \\ -R_{r}^{0}\end{array}\right)$ even in $\mathbf{C}^{(p+m) \times p}$, i.e.,

$$
\operatorname{id}_{p}=\left(\psi D_{l}^{0}\right) S_{r}^{\prime}+N_{l}^{0} R_{r}^{0}=D_{l}^{0}\left(\psi S_{r}^{\prime}\right)+N_{l}^{0} R_{r}^{0}=D_{l}^{0} S_{r}^{0}+N_{l}^{0} R_{r}^{0}, S_{r}^{0}=\psi S_{r}^{\prime} .
$$


Thus $\left(\begin{array}{c}S_{r}^{0} \\ -R_{r}^{0}\end{array}\right)$ is a right inverse of $\left(D_{l}^{0},-N_{l}^{0}\right)$ in $\mathbf{C}^{(p+m) \times p}$ and this can be completed with the matrices from (83), (84), (85) satisfying

$$
\left(\begin{array}{c}
S_{r} \\
-R_{r}
\end{array}\right)=\left(\begin{array}{c}
S_{r}^{0} \\
-R_{r}^{0}
\end{array}\right)-\left(\begin{array}{c}
N_{r}^{0} \\
D_{r}^{0}
\end{array}\right) X,\left(R_{l}, S_{l}\right)=\left(R_{l}^{0}, S_{l}^{0}\right)+X\left(D_{l}^{0},-N_{l}^{0}\right), X \in \mathbf{C}^{m \times p}
$$

and

$$
\left(\begin{array}{cc}
D_{l}^{0} & -N_{l}^{0} \\
R_{l} & S_{l}
\end{array}\right) \in \mathrm{Gl}_{p+m}(\mathbf{C})=\mathrm{Gl}_{N(p+m)}(\mathbf{S}),\left(\begin{array}{cc}
D_{l}^{0} & -N_{l}^{0} \\
R_{l} & S_{l}
\end{array}\right)^{-1}=\left(\begin{array}{cc}
S_{r} & N_{r}^{0} \\
-R_{r} & D_{r}^{0}
\end{array}\right), S_{r}^{0}=\psi S_{r}^{\prime} .
$$

For the robustness assertion of the next Thm. 6.4 we employ the data and results of [16, $\S 2.2]$ and assume that the stability region $\mathbb{D}$ is open. Then $\|d\|:=\max _{z \in \mathbb{C} \backslash \mathbb{D}}|d(z)|$ is a norm on $\mathbf{S}$ and induces maximum norms on $\mathbf{S}^{k \times \ell}$ and $\mathrm{Gl}_{\ell}(\mathbf{S})$. The product of matrices is continuous and $\mathrm{Gl}_{\ell}(\mathbf{S})$ is an open topological subgroup of $\mathbf{S}^{\ell \times \ell}$. A stabilizing tracking controller with matrices $\left(R_{l}, S_{l}\right)$ from Thm. 6.1 is called robust if it is a controller with the same properties for all nearby plants in the just defined norm.

Theorem 6.4. Assume w.l.o.g. that $\psi \in \mathbf{S}$ and that $\left(\psi D_{l}^{0},-N_{l}^{0}\right)$ has a right inverse in $\mathbf{C}^{(p+m) \times p}=\mathbf{S}^{N(p+m) \times N p}$.

(i) Consider the matrices from (118) and complete them to those of (83). Choose a matrix $X \in \mathbf{C}^{m \times p}$ and define the matrices from (119). Then the controller defined by the matrices $\left(R_{l}, S_{l}, R_{r}, S_{r}\right)$ from (119) satisfies the necessary and sufficient condition for tracking and disturbance rejection from Thm. 6.1, i.e., $\psi^{-1} S_{r} \in \mathbf{B}_{\mathbb{D}}^{p \times p}$, if and only if $N_{r}^{0} X \in \mathbf{B}_{\mathbb{D}}^{p \times p} \psi$, for instance if $X \in \mathbf{C}^{m \times p} \psi$.

(ii) Each controller from (i) is robust.

Proof. (i) Since $S_{r}^{0}=\psi S_{r}^{\prime} \in \mathbf{C}^{p \times p} \psi \subset \mathbf{B}_{\mathbb{D}}^{p \times p} \psi$ the assertion follows from $S_{r}=$ $S_{r}^{0}-N_{r}^{0} X$.

(ii) Consider a controller according to (i) and especially the matrices

$$
\left(\begin{array}{cc}
D_{l}^{0} & -N_{l}^{0} \\
R_{l} & S_{l}
\end{array}\right)\left(\begin{array}{cc}
S_{r} & N_{r}^{0} \\
-R_{r} & D_{r}^{0}
\end{array}\right)=\left(\begin{array}{cc}
\operatorname{id}_{p} & 0 \\
0 & \operatorname{id}_{m}
\end{array}\right), S_{r} \in \mathbf{B}_{\mathbb{D}}^{p \times p} \psi, \psi \in \mathbf{S} .
$$

Now consider a plant $\left(\widetilde{D}_{l}^{0},-\widetilde{N}_{l}^{0}\right)$ sufficiently near to $\left(D_{l}^{0},-N_{l}^{0}\right)$. Then

$$
\begin{aligned}
& U:=\left(\widetilde{D}_{l}^{0},-\widetilde{N}_{l}^{0}\right)\left(\begin{array}{c}
S_{r} \\
-R_{r}
\end{array}\right) \operatorname{near}\left(D_{l}^{0},-N_{l}^{0}\right)\left(\begin{array}{c}
S_{r} \\
-R_{r}
\end{array}\right)=\operatorname{id}_{p} \\
& \Longrightarrow U \in \operatorname{Gl}_{N p}(\mathbf{S})=\operatorname{Gl}_{p}(\mathbf{C}),\left(\begin{array}{c}
\widetilde{S}_{r} \\
-\widetilde{R}_{r}
\end{array}\right):=\left(\begin{array}{c}
S_{r} \\
-R_{r}
\end{array}\right) U^{-1} \in \mathbf{C}^{(p+m) \times p}, \\
& \Longrightarrow \operatorname{id}_{p}=\left(\widetilde{D}_{l}^{0},-\widetilde{N}_{l}^{0}\right)\left(\begin{array}{c}
\widetilde{S}_{r} \\
-\widetilde{R}_{r}
\end{array}\right), \widetilde{S}_{r} \in \mathbf{B}_{\mathbb{D}}^{p \times p} \psi .
\end{aligned}
$$

Moreover

$$
\left(R_{l}, S_{l}\right)\left(\begin{array}{c}
\widetilde{S}_{r} \\
-\widetilde{R}_{r}
\end{array}\right)=\left(R_{l}, S_{l}\right)\left(\begin{array}{c}
S_{r} \\
-R_{r}
\end{array}\right) U^{-1}=0
$$

Then there is a unique column $\left(\frac{\widetilde{N_{r}}}{\widetilde{D_{r}}}\right) \in \mathbf{C}^{(p+m) \times m}$ such that

$$
\left(\begin{array}{cc}
\widetilde{D}_{l}^{0} & -\widetilde{N}_{l}^{0} \\
R_{l} & S_{l}
\end{array}\right)\left(\begin{array}{cc}
\widetilde{S}_{r} & \widetilde{N}_{r} \\
-\widetilde{R}_{r} & \widetilde{D}_{r}
\end{array}\right)=\left(\begin{array}{cc}
\operatorname{id}_{p} & 0 \\
0 & \operatorname{id}_{m}
\end{array}\right), \widetilde{S}_{r}=S_{r} U^{-1} \in \mathbf{B}_{\mathbb{D}}^{p \times p} \psi, \psi \in \mathbf{S}
$$

According to Thm. 6.1 the last equation says that the controller with the equation $R_{l} \circ u_{2}+S_{l} \circ y_{2}=0$ is a properly $\mathbb{D}$-stabilizing controller of the plant with the equation $\widetilde{D}_{l}^{0} \circ y_{1}=\widetilde{N}_{l}^{0} \circ u_{1}$ and that this controller tracks signals $r$ and rejects signals 
$u_{1}$ and $u_{2}$ satisfying (95). So the controller $\left(R_{l}, S_{l}\right)$ stabilizes a whole family of plants $\left(\widetilde{D}_{l}^{0},-\widetilde{N}_{l}^{0}\right)$ around the plant $\left(D_{l}^{0},-N_{l}^{0}\right)$ and this is the defining property of robustness.

Lemma 6.5. For the left and right coprime factorizations $G=\left(D_{l}^{0}\right)^{-1} N_{l}^{0}=N_{r}^{0}\left(D_{r}^{0}\right)^{-1}$ over $\mathbf{C}=\mathbf{S}^{N \times N}$ and $\psi \in \mathbf{S}$ the following properties are equivalent:

1. $\left(\psi \mathrm{id}_{p}, N_{l}^{0}\right)$ is right invertible.

2. $\left(\psi \mathrm{id}_{p}, N_{r}^{0}\right)$ is right invertible. .

3. $\left(\psi D_{l}^{0},-N_{l}^{0}\right)$ is right invertible.

Proof. We assume w.l.o.g. that $\psi$ is not a unit. This implies $p \leq m$.

3. $\Longrightarrow 1 .:$ obvious.

1. $\Longrightarrow$ 3.: Consider all primes $\sigma$ of $\mathbf{S}$ and their residue fields $\mathbf{k}(\sigma):=\mathbf{S} / \mathbf{S} \sigma \ni \bar{f}:=$ $f+\mathbf{S} \sigma, f \in \mathbf{S}$. Recall that a matrix $R \in \mathbf{S}^{N p \times \ell}$ is right invertible if and only if $\operatorname{rank}_{\mathbf{k}(\sigma)}(\bar{R})=N p$ for all primes $\sigma$ of $\mathbf{S}$. If 1 . is satisfied we conclude

$$
\begin{aligned}
& \forall \text { primes } \sigma \in \mathbf{S}: N p=\operatorname{rank}_{\mathbf{k}(\sigma)}\left(\overline{D_{l}^{0}},-\overline{N_{l}^{0}}\right)=\operatorname{rank}_{\mathbf{k}(\sigma)}\left(\bar{\psi} \operatorname{id}_{N p}, \overline{N_{l}^{0}}\right) \\
& \Longrightarrow \exists\left(\begin{array}{c}
A \\
B
\end{array}\right) \in \mathbf{k}(\sigma)^{N(p+m) \times N p}: \overline{D_{l}^{0}} A+\overline{N_{l}^{0}} B=\operatorname{id}_{N p} \\
& \Longrightarrow \begin{cases}\text { if } \bar{\psi}=0: & \operatorname{rank}_{\mathbf{k}(\sigma)}\left(\overline{\psi D_{l}^{0}},-\overline{N_{l}^{0}}\right)=\operatorname{rank}_{\mathbf{k}(\sigma)}\left(\bar{\psi} \operatorname{id}_{N p}, \overline{N_{l}^{0}}\right)=N p \\
\text { if } \bar{\psi} \neq 0: & \overline{\psi D_{l}^{0}}\left((\bar{\psi})^{-1} A\right)+\overline{N_{l}^{0}} B=\operatorname{id}_{N p} \Longrightarrow \operatorname{rank}_{\mathbf{k}(\sigma)}\left(\overline{\psi D_{L}^{0}},-\overline{N_{l}^{0}}\right)=N p \\
\Longrightarrow \forall \text { primes } \sigma \in \mathbf{S}: \operatorname{rank}_{\mathbf{k}(q)}\left(\overline{\psi D_{L}^{0}},-\overline{N_{l}^{0}}\right)=N p \Longrightarrow 3 .\end{cases}
\end{aligned}
$$

1. $\Longleftrightarrow 2 .: N_{l}^{0}$ and $N_{r}^{0}$ are equivalent. The equivalence of 1 . and 2. is then shown as that of 1 . and 3 ..

According to [16, Thm. 2 on p. 296] the condition 2. of Lemma 6.5 characterizes the existence of robust compensators in the LTI case and condition 3 . is precisely the assumption of Thm. 6.4.

Corollary 6.6. Assume a controller according to Thm. 6.4,(i), and additionally the $\mathbb{D}$ stable periodic IO-behavior $\mathcal{B}_{2}$ from (109) with the proper transfer matrix $T_{l} \in \mathbf{C}^{m \times p}$. If $\psi^{-1}\left(T_{l}-R_{l}\right) \in \mathbf{B}_{\mathbb{D}}^{m \times p}$ the matrices $\left(R_{l}^{\prime}, S_{l}^{\prime}, T_{l}\right)$ form a robust $(R, S, T)$-controller, cf. $[4,(6.38)]$.

\section{References}

[1] J. Aleixo, J.W. Polderman, P. Rocha, 'Representations and structural properties of periodic systems', Automatica 43(2007), 1921-1931

[2] S. Bittanti, P. Colaneri, Periodic Systems: Filtering and Control, Springer, New York, 2009

[3] I. Blumthaler, U. Oberst, 'Design, parametrization, and pole placement of stabilizing output feedback compensators via injective cogenerator quotient signal modules', Linear Algebra and its Applications 436(2012), 963-1000

[4] H. Bourlès, Linear Systems, ISTE-Wiley, London, 2010 
[5] H. Bourlès, B. Marinescu, U. Oberst, 'The injectivity of the canonical signal module for multidimensional linear systems of difference equations with variable coefficients', Multidimensional Systems and Signal Processing, 2015, DOI 10.1007/s11045-015-0331-x

[6] M.W. Cantoni, K. Glover, 'Gap-metric robustness analysis of linear periodically time-varying feedback systems', SIAM J Control Optimization 38(2000), 803-822

[7] C.T. Chen, Linear System Theory and Design, Harcourt Brace College Publishers, Fort Worth, 1984

[8] G.E. Dullerud, K. Glover, Robust performance of periodic systems, IEEE Transactions on Automatic Control 41(1995), 1146-1159

[9] Y. El Mrabet, H. Bourlès, 'Algebraic Theory of Linear Periodic Discrete-Time Systems in their Polynomial Matrix and Module Descriptions', Proc. 35th Conference on Decision and Control, Kobe, 1996

[10] Y. El Mrabet, H. Bourlès, 'Periodic-polynomial interpretation for structural properties of linear periodic discrete-time systems', Systems and Control Letters 33(1998), 241-251

[11] M. Kuijper, J.C. Willems, 'A behavioral framework for periodically time-varying systems', Proc. 36th IEEE Conference on Decision and Control, San Diego, 1997

[12] T.Y. Lam, Lectures on Modules and Rings, Springer, New York, 1999

[13] J.C. McConnell, J.C. Robson, Noncommutative Noetherian Rings, John Wiley and Sons, Chichester, 1987

[14] A. Quadrat, On a generalization of the Youla-Kučera parametrization. Part II: The lattice approach to MIMO systems, Mathematics of Control Signals Systems 18(2006), 199-235

[15] B. Stenström, Rings of Quotients, Springer, Berlin, 1975

[16] M. Vidyasagar, Control Systems Synthesis, The MIT Press, Cambridge (Ma), 1985

[17] S. Vuglar, M. Cantoni, 'Computing the distance between time-periodic dynamical systems', submitted to MTNS 2016, Minneapolis, July 12-15, 2016

[18] R. Ylinen, 'An algebraic theory for analysis and synthesis of time-varying linear systems', Acta Polytechn Scand. Math. Comp. 32(1980), 1-61 